\title{
On a more accurate half-discrete
}

\section{Hardy-Hilbert-type inequality related to the kernel of exponential function}

Jianquan Liao* and Bicheng Yang

"Correspondence: Imath@163.com Department of Mathematics, Guangdong University of

Education, Guangzhou, Guangdong 51003, P.R. China

\begin{abstract}
By applying the weight functions, the technique of real analysis and Hermite-Hadamard's inequality, a half-discrete Hardy-Hilbert-type inequality related to the kernel of exponential function with the best possible constant factor expressed by the gamma function is given. The more accurate equivalent forms, the operator expressions with the norm, the reverses, and some particular cases are considered.
\end{abstract}

MSC: $26 \mathrm{D} 15$

Keywords: Hardy-Hilbert-type inequality; weight function; equivalent form; reverse; operator

\section{Introduction}

Suppose that $p>1, \frac{1}{p}+\frac{1}{q}=1, f(x), g(y) \geq 0, f \in L^{p}\left(\mathbf{R}_{+}\right), g \in L^{q}\left(\mathbf{R}_{+}\right),\|f\|_{p}=\left(\int_{0}^{\infty} f^{p}(x) d x\right)^{\frac{1}{p}}>$ $0,\|g\|_{q}>0$, and we have the following Hardy-Hilbert integral inequality (cf. [1]):

$$
\int_{0}^{\infty} \int_{0}^{\infty} \frac{f(x) g(y)}{x+y} d x d y<\frac{\pi}{\sin (\pi / p)}\|f\|_{p}\|g\|_{q}
$$

where the constant factor $\frac{\pi}{\sin (\pi / p)}$ is the best possible. If $a_{m}, b_{n} \geq 0, a=\left\{a_{m}\right\}_{m=1}^{\infty} \in l^{p}, b=$ $\left\{b_{n}\right\}_{n=1}^{\infty} \in l^{q},\|a\|_{p}=\left(\sum_{m=1}^{\infty} a_{m}^{p}\right)^{\frac{1}{p}}>0,\|b\|_{q}>0$, then we have the following discrete analogy of (1) with the same best possible constant $\frac{\pi}{\sin (\pi / p)}(c f .[1])$ :

$$
\sum_{m=1}^{\infty} \sum_{n=1}^{\infty} \frac{a_{m} b_{n}}{m+n}<\frac{\pi}{\sin (\pi / p)}\|a\|_{p}\|b\|_{q}
$$

Inequalities (1) and (2) are important in analysis and its applications (cf. [1-5]).

$$
\text { If } \begin{aligned}
\mu_{i}, v_{j} & >0(i, j \in \mathbf{N}=\{1,2, \ldots\}), \\
U_{m} & :=\sum_{i=1}^{m} \mu_{i}, \quad V_{n}:=\sum_{j=1}^{n} v_{j}(m, n \in \mathbf{N}),
\end{aligned}
$$

(0) 2016 Liao and Yang. This article is distributed under the terms of the Creative Commons Attribution 4.0 International License (http://creativecommons.org/licenses/by/4.0/), which permits unrestricted use, distribution, and reproduction in any medium, provided you give appropriate credit to the original author(s) and the source, provide a link to the Creative Commons license, and indicate if changes were made. 
then we have the following Hardy-Hilbert-type inequality (cf. [1], Theorem 321, replacing $\mu_{m}^{1 / q} a_{m}$ and $v_{n}^{1 / p} b_{n}$ by $a_{m}$ and $\left.b_{n}\right)$ :

$$
\sum_{m=1}^{\infty} \sum_{n=1}^{\infty} \frac{a_{m} b_{n}}{U_{m}+V_{n}}<\frac{\pi}{\sin \left(\frac{\pi}{p}\right)}\left(\sum_{m=1}^{\infty} \frac{a_{m}^{p}}{\mu_{m}^{p-1}}\right)^{\frac{1}{p}}\left(\sum_{n=1}^{\infty} \frac{b_{n}^{q}}{v_{n}^{q-1}}\right)^{\frac{1}{q}} .
$$

For $\mu_{i}=v_{j}=1(i, j \in \mathbf{N})$, inequality (4) reduces to (2).

Note The authors of [1] did not prove that (4) is valid with the best possible constant factor.

In 1998, by introducing an independent parameter $\lambda \in(0,1]$, Yang [6] gave an extension of (1) with the kernel $\frac{1}{(x+y)^{\lambda}}$ for $p=q=2$. Following [6], Yang [5] gave some extensions of (1) and (2) as follows:

If $\lambda_{1}, \lambda_{2} \in \mathbf{R}, \lambda_{1}+\lambda_{2}=\lambda, k_{\lambda}(x, y)$ is a non-negative homogeneous function of degree $-\lambda$, with $k\left(\lambda_{1}\right)=\int_{0}^{\infty} k_{\lambda}(t, 1) t^{\lambda_{1}-1} d t \in \mathbf{R}_{+}, \phi(x)=x^{p\left(1-\lambda_{1}\right)-1}, \psi(x)=x^{q\left(1-\lambda_{2}\right)-1}, f(x), g(y) \geq 0$,

$$
f \in L_{p, \phi}\left(\mathbf{R}_{+}\right)=\left\{f ;\|f\|_{p, \phi}:=\left(\int_{0}^{\infty} \phi(x)|f(x)|^{p} d x\right)^{\frac{1}{p}}<\infty\right\}
$$

$g \in L_{q, \psi}\left(\mathbf{R}_{+}\right),\|f\|_{p, \phi},\|g\|_{q, \psi}>0$, then we have

$$
\int_{0}^{\infty} \int_{0}^{\infty} k_{\lambda}(x, y) f(x) g(y) d x d y<k\left(\lambda_{1}\right)\|f\|_{p, \phi}\|g\|_{q, \psi}
$$

where the constant factor $k\left(\lambda_{1}\right)$ is the best possible. Moreover, if $k_{\lambda}(x, y)$ keeps a finite value and $k_{\lambda}(x, y) x^{\lambda_{1}-1}\left(k_{\lambda}(x, y) y^{\lambda_{2}-1}\right)$ is decreasing with respect to $x>0(y>0)$, then, for $a_{m}, b_{n} \geq 0$,

$$
a \in l_{p, \phi}=\left\{a ;\|a\|_{p, \phi}:=\left(\sum_{n=1}^{\infty} \phi(n)\left|a_{n}\right|^{p}\right)^{\frac{1}{p}}<\infty\right\},
$$

$b=\left\{b_{n}\right\}_{n=1}^{\infty} \in l_{q, \psi},\|a\|_{p, \phi},\|b\|_{q, \psi}>0$, we have

$$
\sum_{m=1}^{\infty} \sum_{n=1}^{\infty} k_{\lambda}(m, n) a_{m} b_{n}<k\left(\lambda_{1}\right)\|a\|_{p, \phi}\|b\|_{q, \psi}
$$

where the constant factor $k\left(\lambda_{1}\right)$ is still the best possible.

In 2015, by adding some conditions, Yang [7] gave an extension of (4) as follows:

$$
\begin{aligned}
& \sum_{m=1}^{\infty} \sum_{n=1}^{\infty} \frac{a_{m} b_{n}}{\left(U_{m}+V_{n}\right)^{\lambda}} \\
& \quad<B\left(\lambda_{1}, \lambda_{2}\right)\left(\sum_{m=1}^{\infty} \frac{U_{m}^{p\left(1-\lambda_{1}\right)-1} a_{m}^{p}}{\mu_{m}^{p-1}}\right)^{\frac{1}{p}}\left(\sum_{n=1}^{\infty} \frac{V_{n}^{q\left(1-\lambda_{2}\right)-1} b_{n}^{q}}{v_{n}^{q-1}}\right)^{\frac{1}{q}},
\end{aligned}
$$

where the constant $B\left(\lambda_{1}, \lambda_{2}\right)$ is still the best possible. 
Some other results including multidimensional Hilbert-type inequalities are provided by [8-30].

About the topic of half-discrete Hilbert-type inequalities with the non-homogeneous kernels, Hardy et al. provided a few results in Theorem 351 of [1]. But they did not prove that the constant factors are the best possible. However, Yang [31] gave a result with the kernel $\frac{1}{(1+n x)^{\lambda}}$ by introducing a variable and proved that the constant factor is the best possible. In 2011 Yang [32] gave the following half-discrete Hardy-Hilbert inequality with the best possible constant factor $B\left(\lambda_{1}, \lambda_{2}\right)$ :

$$
\int_{0}^{\infty} f(x)\left[\sum_{n=1}^{\infty} \frac{a_{n}}{(x+n)^{\lambda}}\right] d x<B\left(\lambda_{1}, \lambda_{2}\right)\|f\|_{p, \phi}\|a\|_{q, \psi},
$$

where $\lambda_{1}>0,0<\lambda_{2} \leq 1, \lambda_{1}+\lambda_{2}=\lambda$. Zhong et al. $([17,33,34])$ investigated several halfdiscrete Hilbert-type inequalities with particular kernels. Applying weight functions, a half-discrete Hilbert-type inequality with a general homogeneous kernel of degree $-\lambda \in \mathbf{R}$ and a best constant factor $k\left(\lambda_{1}\right)$ are obtained as follows:

$$
\int_{0}^{\infty} f(x) \sum_{n=1}^{\infty} k_{\lambda}(x, n) a_{n} d x<k\left(\lambda_{1}\right)\|f\|_{p, \phi}\|a\|_{q, \psi}
$$

which is an extension of (8) (cf. [35]). At the same time, a half-discrete Hilbert-type inequality with a general non-homogeneous kernel and a best constant factor are given by Yang [36]. In 2012-2014, Yang et al. published three books [37, 38] and [39] concerned with building the theory of half-discrete Hilbert-type inequalities.

In this paper, by applying weight functions, the technique of real analysis, and HermiteHadamard's inequality, a half-discrete Hardy-Hilbert-type inequality related to the kernel of exponential function with a best possible constant factor expressed by the gamma function is given, which is similar to (7) and an extension of (9) in the following particular kernel:

$$
k_{0}(x, n)=\frac{1}{e^{\alpha\left(\frac{n}{x}\right)^{\gamma}}} \quad(\alpha>0,0<\gamma \leq 1)
$$

Furthermore, the more accurate equivalent forms, the operator expressions with the norm, the reverses, and some particular cases are considered.

\section{An example and some lemmas}

In the following, we agree that $v_{n}>0,0 \leq \tau_{n} \leq \frac{v_{n}}{2}(n \in \mathbf{N}), V_{n}=\sum_{i=1}^{n} v_{i}, \mu(t)$ is a positive continuous function in $\mathbf{R}_{+}=(0, \infty)$,

$$
\begin{gathered}
U(0):=0 ; \quad U(x):=\int_{0}^{x} \mu(t) d t<\infty \quad(x \in(0, \infty)), \\
v(t):=v_{n}, t \in\left(n-\frac{1}{2}, n+\frac{1}{2}\right](n \in \mathbf{N}), \text { and } \\
V\left(\frac{1}{2}\right):=0 ; \quad V(y):=\int_{\frac{1}{2}}^{y} v(t) d t \quad\left(y \in\left(\frac{1}{2}, \infty\right)\right),
\end{gathered}
$$


$p \neq 0,1, \frac{1}{p}+\frac{1}{q}=1, \delta \in\{-1,1\}, f(x), a_{n} \geq 0\left(x \in \mathbf{R}_{+}, n \in \mathbf{N}\right),\|f\|_{p, \Phi_{\delta}}=\left(\int_{0}^{\infty} \Phi_{\delta}(x) f^{p}(x) d x\right)^{\frac{1}{p}}$, $\|a\|_{q, \widehat{\Psi}}=\left(\sum_{n=1}^{\infty} \widehat{\Psi}(n) b_{n}^{q}\right)^{\frac{1}{q}}$, where

$$
\Phi_{\delta}(x):=\frac{U^{p(1-\delta \sigma)-1}(x)}{\mu^{p-1}(x)} \quad\left(x \in \mathbf{R}_{+}\right), \quad \widehat{\Psi}(n):=\frac{\left(V_{n}-\tau_{n}\right)^{q(1-\sigma)-1}}{v_{n}^{q-1}} \quad(n \in \mathbf{N}) .
$$

Example 1 For $\alpha>0,0<\gamma, \sigma \leq 1$, we set $h(t)=\frac{1}{e^{\alpha t \gamma}}\left(t \in \mathbf{R}_{+}\right)$.

(i) Setting $u=\alpha t^{\gamma}$, we find

$$
k(\sigma):=\int_{0}^{\infty} \frac{t^{\sigma-1}}{e^{\alpha t^{\gamma}}} d t=\frac{1}{\gamma \alpha^{\sigma / \gamma}} \int_{0}^{\infty} e^{-u} u^{\frac{\sigma}{\gamma}-1} d u=\frac{\Gamma(\sigma / \gamma)}{\gamma \alpha^{\sigma / \gamma}} \in \mathbf{R}_{+},
$$

where

$$
\Gamma(y):=\int_{0}^{\infty} e^{-v} v^{y-1} d v \quad(y>0)
$$

is called the gamma function (cf. [40]).

(ii) We obtain, for $t>0, \alpha>0,0<\gamma \leq 1, h(t)=\frac{1}{e^{\alpha t \gamma^{\gamma}}}>0, h^{\prime}(t)=-\alpha \gamma t^{\gamma-1} \frac{1}{e^{\alpha t t^{\gamma}}}<0$ and

$$
h^{\prime \prime}(t)=-\alpha \gamma(\gamma-1) t^{\gamma-1} \frac{1}{e^{\alpha t^{\gamma}}}+\left(\alpha \gamma t^{\gamma-1}\right)^{2} \frac{1}{e^{\alpha t^{\gamma}}}>0 .
$$

(iii) If $g(u)>0, g^{\prime}(u)<0, g^{\prime \prime}(u)>0$, then we find that, for $y \in\left(n-\frac{1}{2}, n+\frac{1}{2}\right), g(V(y))>0$, $\frac{d}{d y} g(V(y))=g^{\prime}(V(y)) v_{n}<0$, and

$$
\frac{d^{2}}{d y^{2}} g(V(y))=g^{\prime \prime}(V(y)) v_{n}^{2}>0 \quad(n \in \mathbf{N})
$$

For $g_{1}(u)>0, g_{1}^{\prime}(u)<0, g_{1}^{\prime \prime}(u)>0, g_{2}(u)>0, g_{2}^{\prime}(u) \leq 0, g_{2}^{\prime \prime}(u) \geq 0(u>0)$, we obtain $g_{1}(u) g_{2}(u)>0,\left(g_{1}(u) g_{2}(u)\right)^{\prime}=g_{1}^{\prime}(u) g_{2}(u)+g_{1}(u) g_{2}^{\prime}(u)<0$, and

$$
\left(g_{1}(u) g_{2}(u)\right)^{\prime \prime}=g_{1}^{\prime \prime}(u) g_{2}(u)+2 g_{1}^{\prime}(u) g_{2}^{\prime}(u)+g_{1}(u) g_{2}^{\prime \prime}(u)>0 \quad(u>0) .
$$

(iv) For $\alpha>0,0<\gamma, \sigma \leq 1, c>0$, we have $h(c V(y)) V^{\sigma-1}(y)>0, \frac{d}{d y}\left(h(c V(y)) V^{\sigma-1}(y)\right)<0$, and

$$
\frac{d^{2}}{d y^{2}}\left(h(c V(y)) V^{\sigma-1}(y)\right)>0 \quad\left(y \in\left(n-\frac{1}{2}, n+\frac{1}{2}\right), n \in \mathbf{N}\right) .
$$

Then by Hermite-Hadamard's inequality ( $c f$. [41]), we have

$$
h(c V(n)) V^{\sigma-1}(n)<\int_{n-\frac{1}{2}}^{n+\frac{1}{2}} h(c V(y)) V^{\sigma-1}(y) d y \quad(n \in \mathbf{N}) .
$$

Lemma 1 If $g(t)(>0)$ is a strictly decreasing continuous function in $\left(\frac{1}{2}, \infty\right)$, which is strictly convex satisfying $\int_{\frac{1}{2}}^{\infty} g(t) d t \in \mathbf{R}_{+}$, then we have

$$
\int_{1}^{\infty} g(t) d t<\sum_{n=1}^{\infty} g(n)<\int_{\frac{1}{2}}^{\infty} g(t) d t
$$


Proof By Hermite-Hadamard's inequality and the decreasing property, we have

$$
\int_{n}^{n+1} g(t) d t<\int_{n}^{n+1} g(n) d t=g(n)<\int_{n-\frac{1}{2}}^{n+\frac{1}{2}} g(t) d t \quad(n \in \mathbf{N})
$$

and, for $n_{0} \in \mathbf{N}$, it follows that

$$
\begin{aligned}
& \int_{1}^{n_{0}+1} g(t) d t<\sum_{n=1}^{n_{0}} g(n)<\sum_{n=1}^{n_{0}} \int_{n-\frac{1}{2}}^{n+\frac{1}{2}} g(t) d t=\int_{\frac{1}{2}}^{n_{0}+\frac{1}{2}} g(t) d t, \\
& \int_{n_{0}+1}^{\infty} g(t) d t \leq \sum_{n=n_{0}+1}^{\infty} g(n) \leq \int_{n_{0}+\frac{1}{2}}^{\infty} g(t) d t<\infty .
\end{aligned}
$$

Hence, choosing plus for the above two inequalities, we have (12).

Lemma 2 If $\alpha>0,0<\gamma, \sigma \leq 1$, define the following weight coefficients:

$$
\begin{aligned}
& \omega_{\delta}(\sigma, x):=\sum_{n=1}^{\infty} \frac{1}{e^{\alpha U^{\delta \gamma}(x)\left(V_{n}-\tau_{n}\right)^{\gamma}}} \frac{U^{\delta \sigma}(x) v_{n}}{\left(V_{n}-\tau_{n}\right)^{1-\sigma}}, \quad x \in \mathbf{R}_{+}, \\
& \varpi_{\delta}(\sigma, n):=\int_{0}^{\infty} \frac{1}{e^{\alpha U^{\delta \gamma}(x)\left(V_{n}-\tau_{n}\right)^{\gamma}}} \frac{\left(V_{n}-\tau_{n}\right)^{\sigma} \mu(x)}{U^{1-\delta \sigma}(x)} d x, \quad n \in \mathbf{N} .
\end{aligned}
$$

Then we have the following inequalities:

$$
\begin{aligned}
& \omega_{\delta}(\sigma, x)<k(\sigma) \quad\left(x \in \mathbf{R}_{+}\right), \\
& \varpi_{\delta}(\sigma, n) \leq k(\sigma) \quad(n \in \mathbf{N}),
\end{aligned}
$$

where $k(\sigma)$ is indicated by (10).

Proof Since $V_{n}-\tau_{n} \geq \int_{\frac{1}{2}}^{n+\frac{1}{2}} v(t) d t-\frac{v_{n}}{2}=\int_{\frac{1}{2}}^{n} v(t) d t=V(n)$, and, for $t \in\left(n-\frac{1}{2}, n+\frac{1}{2}\right), v_{n}=$ $V^{\prime}(t)$, by (11) (for $\left.c=U^{\delta}(x)\right)$ and (12), we have

$$
\begin{aligned}
& \frac{1}{e^{\alpha U^{\delta \gamma}(x)\left(V_{n}-\tau_{n}\right)^{\gamma}} \frac{U^{\delta \sigma}(x)}{\left(V_{n}-\tau_{n}\right)^{1-\sigma}}} \leq \frac{1}{e^{\alpha L^{\delta \gamma}(x) V^{\gamma}(n)}} \frac{U^{\delta \sigma}(x)}{V^{1-\sigma}(n)} \\
& \quad<\int_{n-\frac{1}{2}}^{n+\frac{1}{2}} \frac{1}{e^{\alpha U^{\delta \gamma}(x) V^{\gamma}(t)}} \frac{U^{\delta \sigma}(x)}{V^{1-\sigma}(t)} d t \quad(n \in \mathbf{N}), \\
& \omega_{\delta}(\sigma, x)<\sum_{n=1}^{\infty} v_{n} \int_{n-\frac{1}{2}}^{n+\frac{1}{2}} \frac{1}{e^{\alpha U^{\delta \gamma}(x) V \gamma(t)}} \frac{U^{\delta \sigma}(x)}{V^{1-\sigma}(t)} d t \\
& =\sum_{n=1}^{\infty} \int_{n-\frac{1}{2}}^{n+\frac{1}{2}} \frac{1}{e^{\alpha L^{\delta \gamma}(x) V^{\gamma}(t)}} \frac{U^{\delta \sigma}(x) V^{\prime}(t)}{V^{1-\sigma}(t)} d t \\
& =\int_{\frac{1}{2}}^{\infty} \frac{1}{e^{\alpha U^{\delta \gamma}(x) V^{\gamma}(t)}} \frac{U^{\delta \sigma}(x) V^{\prime}(t)}{V^{1-\sigma}(t)} d t .
\end{aligned}
$$


Setting $u=U^{\delta}(x) V(t)$, by (10), we find

$$
\begin{aligned}
\omega_{\delta}(\sigma, x) & <\int_{U^{\delta}(x) V\left(\frac{1}{2}\right)}^{U^{\delta}(x) V(\infty)} \frac{1}{e^{\alpha u^{\gamma}}} \frac{U^{\delta \sigma}(x) U^{-\delta}(x)}{\left(u U^{-\delta}(x)\right)^{1-\sigma}} d u \\
& \leq \int_{0}^{\infty} \frac{1}{e^{\alpha u^{\gamma}}} u^{\sigma-1} d u=k(\sigma) .
\end{aligned}
$$

Hence, (16) follows.

Setting $u=\left(V_{n}-\tau_{n}\right) U^{\delta}(x)$ in (15), we find $d u=\delta\left(V_{n}-\tau_{n}\right) U^{\delta-1}(x) \mu(x) d x$ and

$$
\varpi_{\delta}(\sigma, n)=\frac{1}{\delta} \int_{\left(V_{n}-\tau_{n}\right) U^{\delta}(0)}^{\left(V_{n}-\tau_{n}\right) U^{\delta}(\infty)} \frac{1}{e^{\alpha u^{\gamma}}} u^{\sigma-1} d u
$$

If $\delta=1$, then

$$
\varpi_{1}(\sigma, n)=\int_{0}^{\left(V_{n}-\tau_{n}\right) U(\infty)} \frac{1}{e^{\alpha u^{\gamma}}} u^{\sigma-1} d u \leq \int_{0}^{\infty} \frac{1}{e^{\alpha u^{\gamma}}} u^{\sigma-1} d u
$$

if $\delta=-1$, then

$$
\varpi_{-1}(\sigma, n)=-\int_{\infty}^{\left(V_{n}-\tau_{n}\right) U^{-1}(\infty)} \frac{1}{e^{\alpha u^{\gamma}}} u^{\sigma-1} d u \leq \int_{0}^{\infty} \frac{1}{e^{\alpha u^{\gamma}}} u^{\sigma-1} d u
$$

Hence, by (10), we have (17).

Remark 1 (i) We do not need the condition of $\sigma \leq 1$ in obtaining (17). (ii) If $U(\infty)=\infty$, then we have

$$
\varpi_{\delta}(\sigma, n)=k(\sigma) \quad(n \in \mathbf{N}) .
$$

For example, we set $\mu(t)=\frac{1}{(1+t)^{a}}(t>0 ; 0 \leq a \leq 1)$, then for $x \geq 0$, we find

$$
U(x)=\int_{0}^{x} \frac{d t}{(1+t)^{a}}= \begin{cases}\frac{(1+x)^{1-a}-1}{1-a}, & 0 \leq a<1,<\infty, \\ \ln (1+x), & a=1\end{cases}
$$

$U(0)=0$, and $U(\infty)=\int_{0}^{\infty} \frac{d t}{(1+t)^{a}}=\infty$.

Lemma 3 If $\alpha>0,0<\gamma, \sigma \leq 1$, there exists $n_{0} \in \mathbf{N}$, such that $\left\{v_{n}\right\}_{n=n_{0}}^{\infty}$ is decreasing and $V(\infty)=\infty$, then: (i) for $x \in \mathbf{R}_{+}$, we have

$$
k(\sigma)\left(1-\theta_{\delta}(\sigma, x)\right)<\omega_{\delta}(\sigma, x)
$$

where

$$
\theta_{\delta}(\sigma, x):=\frac{1}{k(\sigma)} \int_{0}^{U^{\delta}(x) V\left(n_{0}+1\right)} \frac{u^{\sigma-1}}{e^{\alpha u^{\gamma}}} d u=O\left((U(x))^{\delta \sigma}\right) \in(0,1)
$$


(ii) for any $b>0$, we have

$$
\sum_{n=1}^{\infty} \frac{v_{n}}{\left(V_{n}-\tau_{n}\right)^{1+b}}=\frac{1}{b}\left(\frac{1}{v_{1}^{b}}+b O(1)\right)
$$

Proof Since $V_{n}-\tau_{n} \leq V_{n} \leq V_{n+1}-\frac{v_{n+1}}{2}=V(n+1)$, and $v_{n} \geq V^{\prime}(t)\left(t \in(n, n+1) ; n \geq n_{0}\right)$, by (13), we find

$$
\begin{aligned}
\omega_{\delta}(\sigma, x) & \geq \sum_{n=n_{0}}^{\infty} \frac{1}{e^{\alpha U^{\delta \gamma}(x) V^{\gamma}(n+1)}} \frac{U^{\delta \sigma}(x) v_{n+1}}{V^{1-\sigma}(n+1)} \\
& =\sum_{n=n_{0}+1}^{\infty} \frac{1}{e^{\alpha U^{\delta \gamma}(x) V^{\gamma}(n)}} \frac{U^{\delta \sigma}(x) v_{n}}{V^{1-\sigma}(n)} \\
& >\sum_{n=n_{0}+1}^{\infty} \int_{n}^{n+1} \frac{1}{e^{\alpha U^{\delta \gamma}(x) V^{\gamma}(t)}} \frac{U^{\delta \sigma}(x) V^{\prime}(t)}{V^{1-\sigma}(t)} d t \\
& =\int_{n_{0}+1}^{\infty} \frac{1}{e^{\alpha U^{\delta \gamma}(x) V \gamma(t)}} \frac{U^{\delta \sigma}(x) V^{\prime}(t)}{V^{1-\sigma}(t)} d t .
\end{aligned}
$$

Setting $u=U^{\delta}(x) V(t)$, in view of $V(\infty)=\infty$, by (10), we find

$$
\omega_{\delta}(\sigma, x)>\int_{U^{\delta}(x) V\left(n_{0}+1\right)}^{\infty} \frac{u^{\sigma-1}}{e^{\alpha u^{\gamma}}} d u=k(\sigma)-\int_{0}^{U^{\delta}(x) V\left(n_{0}+1\right)} \frac{u^{\sigma-1}}{e^{\alpha u^{\gamma}}} d u=k(\sigma)\left(1-\theta_{\delta}(\sigma, x)\right) .
$$

We find

$$
0<\theta_{\delta}(\sigma, x) \leq \frac{1}{k(\sigma)} \int_{0}^{U^{\delta}(x) V\left(n_{0}+1\right)} u^{\sigma-1} d u=\frac{\left(U^{\delta}(x) V\left(n_{0}+1\right)\right)^{\sigma}}{\sigma k(\sigma)} \quad\left(x \in \mathbf{R}_{+}\right)
$$

and then (19) follows.

For $b>0$, we find

$$
\begin{aligned}
\sum_{n=1}^{\infty} \frac{v_{n}}{\left(V_{n}-\tau_{n}\right)^{1+b}} & \leq \sum_{n=1}^{\infty} \frac{v_{n}}{V^{1+b}(n)}=\frac{v_{1}}{V^{1+b}(1)}+\sum_{n=2}^{\infty} \frac{v_{n}}{V^{1+b}(n)} \\
& <\frac{2^{1+b}}{v_{1}^{b}}+\sum_{n=2}^{\infty} \int_{n-\frac{1}{2}}^{n+\frac{1}{2}} \frac{V^{\prime}(x) d x}{V^{1+b}(x)}=\frac{2^{1+b}}{v_{1}^{b}}+\int_{\frac{3}{2}}^{\infty} \frac{V^{\prime}(x) d x}{V^{1+b}(x)} \\
& =\frac{2^{1+b}}{v_{1}^{b}}+\frac{v_{1}^{-b}}{b}=\frac{1}{b}\left(\frac{1}{v_{1}^{b}}+b \frac{2^{1+b}}{v_{1}^{b}}\right) \\
\sum_{n=1}^{\infty} \frac{v_{n}}{\left(V_{n}-\tau_{n}\right)^{1+b}} & \geq \sum_{n=n_{0}}^{\infty} \frac{v_{n}}{\left(V_{n}-\tau_{n}\right)^{1+b}} \geq \sum_{n=n_{0}}^{\infty} \frac{v_{n+1}}{V^{1+b}(n+1)} \\
& =\sum_{n=n_{0}+1}^{\infty} \frac{v_{n}}{V^{1+b}(n)}>\sum_{n=n_{0}+1}^{\infty} \int_{n}^{n+1} \frac{V^{\prime}(x) d x}{V^{1+b}(x)}=\int_{n_{0}+1}^{\infty} \frac{V^{\prime}(x) d x}{V^{1+b}(x)} \\
& =\frac{1}{b V^{b}\left(n_{0}+1\right)}=\frac{1}{b}\left(\frac{1}{v_{1}^{b}}+b \frac{V^{-b}\left(n_{0}+1\right)-v_{1}^{-b}}{b}\right) .
\end{aligned}
$$

Since $\frac{V^{-b}\left(n_{0}+1\right)-v_{1}^{-b}}{b} \rightarrow$ Constant $\left(b \rightarrow 0^{+}\right)$, we have (20). 
Note For example, $v_{n}=\frac{1}{n^{a}}(n \in \mathbf{N} ; 0 \leq a \leq 1)$ satisfies the conditions of $\left\{v_{n}\right\}_{n=1}^{\infty}$ in Lemma 3 (for $n_{0}=1$ ).

\section{Main results and operator expressions}

Theorem 1 If $\alpha>0,0<\gamma, \sigma \leq 1$, then for $p>1,0<\|f\|_{p, \Phi_{\delta}},\|a\|_{q, \widehat{\Psi}}<\infty$, we have the following equivalent inequalities:

$$
\begin{aligned}
& I:=\sum_{n=1}^{\infty} \int_{0}^{\infty} \frac{a_{n} f(x)}{e^{\alpha U^{\delta \gamma}(x)\left(V_{n}-\tau_{n}\right)^{\gamma}}} d x<\frac{\Gamma(\sigma / \gamma)}{\gamma \alpha^{\sigma / \gamma}}\|f\|_{p, \Phi_{\delta}}\|a\|_{q, \widehat{\Psi}}, \\
& J_{1}:=\sum_{n=1}^{\infty} \frac{v_{n}}{\left(V_{n}-\tau_{n}\right)^{1-p \sigma}}\left[\int_{0}^{\infty} \frac{f(x) d x}{e^{\alpha U^{\delta \gamma}(x)\left(V_{n}-\tau_{n}\right)^{\gamma}}}\right]^{p}<\frac{\Gamma(\sigma / \gamma)}{\gamma \alpha^{\sigma / \gamma}}\|f\|_{p, \Phi_{\delta}}, \\
& J_{2}:=\left\{\int_{0}^{\infty} \frac{\mu(x)}{U^{1-q \delta \sigma}(x)}\left[\sum_{n=1}^{\infty} \frac{a_{n}}{e^{\alpha U^{\delta \gamma}(x)\left(V_{n}-\tau_{n}\right)^{\gamma}}}\right]^{q} d x\right\}^{\frac{1}{q}}<\frac{\Gamma(\sigma / \gamma)}{\gamma \alpha^{\sigma / \gamma}}\|a\|_{q, \widehat{\Psi}} .
\end{aligned}
$$

Proof By Hölder's inequality with weight (cf. [41]), we have

$$
\begin{aligned}
& {\left[\int_{0}^{\infty} \frac{f(x)}{e^{\alpha U^{\delta \gamma}(x)\left(V_{n}-\tau_{n}\right)^{\gamma}}} d x\right]^{p}} \\
& =\left[\int_{0}^{\infty} \frac{1}{e^{\alpha U^{\delta \gamma}(x)\left(V_{n}-\tau_{n}\right)^{\gamma}}} \frac{U^{\frac{1-\delta \sigma}{q}}(x) f(x)}{\left(V_{n}-\tau_{n}\right)^{\frac{1-\sigma}{p}}} \mu^{\frac{1}{q}}(x) \frac{\left(V_{n}-\tau_{n}\right)^{\frac{1-\sigma}{p}} \mu^{\frac{1}{q}}(x)}{U^{\frac{1-\delta \sigma}{q}}(x)} d x\right]^{p} \\
& \leq \int_{0}^{\infty} \frac{\left(V_{n}-\tau_{n}\right)^{\gamma}}{e^{\alpha U^{\delta \gamma}(x)\left(V_{n}-\tau_{n}\right)^{\gamma}}}\left[\frac{U^{\frac{p(1-\delta \sigma)}{q}}(x) f^{p}(x)}{\left(V_{n}-\tau_{n}\right)^{1-\sigma} \mu^{\frac{p}{q}}(x)}\right] d x \\
& \times\left[\int_{0}^{\infty} \frac{\left(V_{n}-\tau_{n}\right)^{\gamma}}{e^{\alpha U^{\delta \gamma}(x)\left(V_{n}-\tau_{n}\right)^{\gamma}}} \frac{\left(V_{n}-\tau_{n}\right)^{(1-\sigma)(p-1)} \mu(x)}{U^{1-\delta \sigma}(x)} d x\right]^{p-1} \\
& =\frac{\left(\varpi_{\delta}(\sigma, n)\right)^{p-1}}{\left(V_{n}-\tau_{n}\right)^{p \sigma-1} v_{n}} \int_{0}^{\infty} \frac{1}{e^{\alpha U^{\delta \gamma}(x)\left(V_{n}-\tau_{n}\right)^{\gamma}}} \frac{U^{(1-\delta \sigma)(p-1)}(x) \nu_{n} f^{p}(x)}{\left(V_{n}-\tau_{n}\right)^{1-\sigma} \mu^{p-1}(x)} d x .
\end{aligned}
$$

In view of (17) and the Lebesgue term by term integration theorem (cf. [42]), we find

$$
\begin{aligned}
J_{1} & \leq(k(\sigma))^{\frac{1}{q}}\left[\sum_{n=1}^{\infty} \int_{0}^{\infty} \frac{1}{e^{\alpha U^{\delta \gamma}(x)\left(V_{n}-\tau_{n}\right)^{\gamma}}} \frac{U^{(1-\delta \sigma)(p-1)}(x) v_{n}}{\left(V_{n}-\tau_{n}\right)^{1-\sigma} \mu^{p-1}(x)} f^{p}(x) d x\right]^{\frac{1}{p}} \\
& =(k(\sigma))^{\frac{1}{q}}\left[\int_{0}^{\infty} \sum_{n=1}^{\infty} \frac{1}{e^{\alpha U^{\delta \gamma}(x)\left(V_{n}-\tau_{n}\right)^{\gamma}}} \frac{U^{(1-\delta \sigma)(p-1)}(x) v_{n}}{\left(V_{n}-\tau_{n}\right)^{1-\sigma} \mu^{p-1}(x)} f^{p}(x) d x\right]^{\frac{1}{p}} \\
& =(k(\sigma))^{\frac{1}{q}}\left[\int_{0}^{\infty} \omega_{\delta}(\sigma, x) \frac{U^{p(1-\delta \sigma)-1}(x)}{\mu^{p-1}(x)} f^{p}(x) d x\right]^{\frac{1}{p}} .
\end{aligned}
$$

Then by (16), we have (22).

By Hölder's inequality (cf. [41]), we have

$$
\begin{aligned}
I & =\sum_{n=1}^{\infty}\left[\frac{v_{n}^{\frac{1}{p}}}{\left(V_{n}-\tau_{n}\right)^{\frac{1}{p}-\sigma}} \int_{0}^{\infty} \frac{f(x)}{e^{\alpha U^{\delta \gamma}(x)\left(V_{n}-\tau_{n}\right)^{\gamma}}} d x\right]\left[\frac{\left(V_{n}-\tau_{n}\right)^{\frac{1}{p}-\sigma} a_{n}}{v_{n}^{1 / p}}\right] \\
& \leq J_{1}\|a\|_{q, \widehat{\Psi}} .
\end{aligned}
$$


Then by (22), we have (21). On the other hand, assuming that (21) is valid, we set

$$
a_{n}:=\frac{v_{n}}{\left(V_{n}-\tau_{n}\right)^{1-p \sigma}}\left[\int_{0}^{\infty} \frac{1}{e^{\alpha U^{\delta \gamma}(x)\left(V_{n}-\tau_{n}\right)^{\gamma}}} f(x) d x\right]^{p-1}, \quad n \in \mathbf{N}
$$

Then we find $J_{1}^{p}=\|a\|_{q, \widehat{\Psi}}^{q}$. If $J_{1}=0$, then (22) is trivially valid; if $J_{1}=\infty$, then (22) remains impossible. Suppose that $0<J_{1}<\infty$. By (21), we have

$$
\begin{aligned}
& \|a\|_{q, \widehat{\Psi}}^{q}=J_{1}^{p}=I<k(\sigma)\|f\|_{p, \Phi_{\delta}}\|a\|_{q, \widehat{\Psi}}, \\
& \|a\|_{q, \widehat{\Psi}}^{q-1}=J_{1}<k(\sigma)\|f\|_{p, \Phi_{\delta}},
\end{aligned}
$$

and then (22) follows, which is equivalent to (21).

Still by Hölder's inequality with weight ( $c f$. [41]), we have

$$
\begin{aligned}
& {\left[\sum_{n=1}^{\infty} \frac{a_{n}}{e^{\alpha U^{\delta \gamma}(x)\left(V_{n}-\tau_{n}\right)^{\gamma}}}\right]^{q} } \\
&=\left[\sum_{n=1}^{\infty} \frac{1}{e^{\alpha U^{\delta \gamma}(x)\left(V_{n}-\tau_{n}\right)^{\gamma}}} \cdot \frac{U^{\frac{1-\delta \sigma}{q}}(x) v_{n}^{\frac{1}{p}}}{\left(V_{n}-\tau_{n}\right)^{\frac{1-\sigma}{p}}} \cdot \frac{\left(V_{n}-\tau_{n}\right)^{\frac{1-\sigma}{p}} a_{n}}{U^{\frac{1-\delta \sigma}{q}}(x) v_{n}^{1 / p}}\right]^{q} \\
& \leq {\left[\sum_{n=1}^{\infty} \frac{1}{e^{\alpha U^{\delta \gamma}(x)\left(V_{n}-\tau_{n}\right)^{\gamma}}} \frac{U^{(1-\delta \sigma)(p-1)}(x) v_{n}}{\left(V_{n}-\tau_{n}\right)^{1-\sigma}}\right]^{q-1} } \\
& \times \sum_{n=1}^{\infty} \frac{1}{e^{\alpha U^{\delta \gamma}(x)\left(V_{n}-\tau_{n}\right)^{\gamma}}} \frac{\left(V_{n}-\tau_{n}\right)^{\frac{q(1-\sigma)}{p}}}{U^{1-\delta \sigma}(x) v_{n}^{q-1}} a_{n}^{q} \\
&= \frac{\left(\omega_{\delta}(\sigma, x)\right)^{q-1}}{U^{q \delta \sigma-1}(x) \mu(x)} \sum_{n=1}^{\infty} \frac{1}{e^{\alpha U^{\delta \gamma}(x)\left(V_{n}-\tau_{n}\right)^{\gamma}}} \frac{\left(V_{n}-\tau_{n}\right)^{(1-\sigma)(q-1)} \mu(x)}{U^{1-\delta \sigma}(x) v_{n}^{q-1}} a_{n}^{q} .
\end{aligned}
$$

Then by (16) and the Lebesgue term by term integration theorem ( $c f$. [42]), it follows that

$$
\begin{aligned}
J_{2} & <(k(\sigma))^{\frac{1}{p}}\left[\int_{0}^{\infty} \sum_{n=1}^{\infty} \frac{1}{e^{\alpha U^{\delta \gamma}(x)\left(V_{n}-\tau_{n}\right)^{\gamma}}} \frac{\left(V_{n}-\tau_{n}\right)^{(1-\sigma)(q-1)} \mu(x)}{U^{1-\delta \sigma}(x) v_{n}^{q-1}} a_{n}^{q} d x\right]^{\frac{1}{q}} \\
& =(k(\sigma))^{\frac{1}{p}}\left[\sum_{n=1}^{\infty} \int_{0}^{\infty} \frac{1}{e^{\alpha U^{\delta \gamma}(x)\left(V_{n}-\tau_{n}\right)^{\gamma}}} \frac{\left(V_{n}-\tau_{n}\right)^{(1-\sigma)(q-1)} \mu(x)}{U^{1-\delta \sigma}(x) v_{n}^{q-1}} a_{n}^{q} d x\right]^{\frac{1}{q}} \\
& =(k(\sigma))^{\frac{1}{p}}\left[\sum_{n=1}^{\infty} \varpi_{\delta}(\sigma, n) \frac{\left(V_{n}-\tau_{n}\right)^{q(1-\sigma)-1}}{v_{n}^{q-1}} a_{n}^{q}\right]^{\frac{1}{q}} .
\end{aligned}
$$

Then by (17), we have (23).

By Hölder's inequality (cf. [41]), we have

$$
\begin{aligned}
I & =\int_{0}^{\infty}\left(\frac{U^{\frac{1}{q}-\delta \sigma}(x)}{\mu^{\frac{1}{q}}(x)} f(x)\right)\left[\frac{\mu^{\frac{1}{q}}(x)}{U^{\frac{1}{q}-\delta \sigma}(x)} \sum_{n=1}^{\infty} \frac{1}{e^{\alpha U^{\delta \gamma}(x)\left(V_{n}-\tau_{n}\right)^{\gamma}}} a_{n}\right] d x \\
& \leq\|f\|_{p, \Phi_{\delta}} J_{2} .
\end{aligned}
$$


Then by (23), we have (21). On the other hand, assuming that (23) is valid, we set

$$
f(x):=\frac{\mu(x)}{U^{1-q \delta \sigma}(x)}\left[\sum_{n=1}^{\infty} \frac{1}{e^{\alpha U^{\delta \gamma}(x)\left(V_{n}-\tau_{n}\right)^{\gamma}}} a_{n}\right]^{q-1}, \quad x \in \mathbf{R}_{+} .
$$

Then we find $J_{2}^{q}=\|f\|_{p, \Phi_{\delta}}^{p}$. If $J_{2}=0$, then (23) is trivially valid; if $J_{2}=\infty$, then (23) keeps impossible. Suppose that $0<J_{2}<\infty$. By (21), we have

$$
\|f\|_{p, \Phi_{\delta}}^{p}=J_{2}^{q}=I<k(\sigma)\|f\|_{p, \Phi_{\delta}}\|a\|_{q, \widehat{\Psi}}, \quad\|f\|_{p, \Phi_{\delta}}^{p-1}=J_{2}<k(\sigma)\|a\|_{q, \widehat{\Psi}},
$$

and then (23) follows, which is equivalent to (21).

Therefore, (21), (22), and (23) are equivalent.

Theorem 2 As regards the assumptions of Theorem 1, if there exists $n_{0} \in \mathbf{N}$, such that $\left\{v_{n}\right\}_{n=n_{0}}^{\infty}$ is decreasing and $U(\infty)=V(\infty)=\infty$, then the constant factor $k(\sigma)=\frac{\Gamma(\sigma / \gamma)}{\gamma \alpha^{\sigma / \gamma}}$ in (21), (22), and (23) is the best possible.

Proof For $\varepsilon \in(0, q \sigma)$, we set $\widetilde{\sigma}=\sigma-\frac{\varepsilon}{q}(\in(0,1))$, and $\widetilde{f}=\tilde{f}(x), x \in \mathbf{R}_{+}, \tilde{a}=\left\{\tilde{a}_{n}\right\}_{n=1}^{\infty}$,

$$
\begin{aligned}
& \tilde{f}(x)= \begin{cases}U^{\delta(\widetilde{\sigma}+\varepsilon)-1}(x) \mu(x), & 0<x^{\delta} \leq 1, \\
0, & x^{\delta}>0,\end{cases} \\
& \tilde{a}_{n}=\left(V_{n}-\tau_{n}\right)^{\widetilde{\sigma}-1} v_{n}=\left(V_{n}-\tau_{n}\right)^{\sigma-\frac{\varepsilon}{q}-1} v_{n}, \quad n \in \mathbf{N} .
\end{aligned}
$$

Then for $\delta= \pm 1$, since $U(\infty)=\infty$, we find

$$
\int_{\left\{x>0 ; 0<x^{\delta} \leq 1\right\}} \frac{\mu(x)}{U^{1-\delta \varepsilon}(x)} d x=\frac{1}{\varepsilon} U^{\delta \varepsilon}(1) .
$$

By (20), (32), and (19), we obtain

$$
\begin{aligned}
\|\widetilde{f}\|_{p, \Phi_{\delta}}\|\widetilde{a}\|_{q, \Psi} & =\left(\int_{\left\{x>0 ; 0<x^{\delta} \leq 1\right\}} \frac{\mu(x) d x}{U^{1-\delta \varepsilon}(x)}\right)^{\frac{1}{p}}\left[\sum_{n=1}^{\infty} \frac{v_{n}}{\left(V_{n}-\tau_{n}\right)^{1+\varepsilon}}\right]^{\frac{1}{q}} \\
& =\frac{1}{\varepsilon} U^{\frac{\delta \varepsilon}{p}}(1)\left(\frac{1}{v_{1}^{\varepsilon}}+\varepsilon O(1)\right)^{\frac{1}{q}}, \\
\widetilde{I} & :=\int_{0}^{\infty} \sum_{n=1}^{\infty} \frac{1}{e^{\alpha U^{\delta \gamma}(x)\left(V_{n}-\tau_{n}\right)^{\gamma}} \widetilde{a}_{n} \widetilde{f}(x) d x} \\
& =\int_{\left\{x>0 ; 0<x^{\delta} \leq 1\right\}} \sum_{n=1}^{\infty} \frac{1}{e^{\alpha U^{\delta \gamma}(x)\left(V_{n}-\tau_{n}\right)^{\gamma}}} \frac{\left(V_{n}-\tau_{n}\right)^{\tilde{\sigma}-1} v_{n} \mu(x)}{U^{1-\delta(\widetilde{\sigma}+\varepsilon)}(x)} d x \\
& =\int_{\left\{x>0 ; 0<x^{\delta} \leq 1\right\}} \omega_{\delta}(\widetilde{\sigma}, x) \frac{\mu(x)}{U^{1-\delta \varepsilon}(x)} d x \\
& \geq k(\widetilde{\sigma}) \int_{\left\{x>0 ; 0<x^{\delta} \leq 1\right\}}\left(1-\theta_{\delta}(\widetilde{\sigma}, x)\right) \frac{\mu(x)}{U^{1-\delta \varepsilon}(x)} d x \\
& =k(\widetilde{\sigma}) \int_{\left\{x>0 ; 0<x^{\delta} \leq 1\right\}}\left(1-O\left((U(x))^{\delta \widetilde{\sigma}}\right)\right) \frac{\mu(x)}{U^{1-\delta \varepsilon}(x)} d x
\end{aligned}
$$




$$
\begin{aligned}
& =k(\widetilde{\sigma})\left[\int_{\left\{x>0 ; 0<x^{\delta} \leq 1\right\}} \frac{\mu(x) d x}{U^{1-\delta \varepsilon}(x)}-\int_{\left\{x>0 ; 0<x^{\delta} \leq 1\right\}} O\left(\frac{\mu(x)}{U^{1-\delta\left(\sigma+\frac{\varepsilon}{p}\right)}(x)}\right) d x\right] \\
& =\frac{1}{\varepsilon} k\left(\sigma-\frac{\varepsilon}{q}\right)\left(U^{\delta \varepsilon}(1)-\varepsilon O_{1}(1)\right) .
\end{aligned}
$$

If there exists a positive constant $K \leq k(\sigma)$, such that (21) is valid when replacing $k(\sigma)$ to $K$, then in particular, by Lebesgue term by term integration theorem, we have $\varepsilon \widetilde{I}<\varepsilon K\|\tilde{f}\|_{p, \Phi_{\delta}}\|\widetilde{a}\|_{q, \Psi}$, namely,

$$
k\left(\sigma-\frac{\varepsilon}{q}\right)\left(U^{\delta \varepsilon}(1)-\varepsilon O_{1}(1)\right)<K \cdot U^{\frac{\delta \varepsilon}{p}}(1)\left(\frac{1}{\nu_{1}^{\varepsilon}}+\varepsilon O(1)\right)^{\frac{1}{q}} .
$$

It follows that $k(\sigma) \leq K\left(\varepsilon \rightarrow 0^{+}\right)$. Hence, $K=k(\sigma)$ is the best possible constant factor of (21).

The constant factor $k(\sigma)$ in (22) ((23)) is still the best possible. Otherwise, we would reach a contradiction by (26) ((29)) that the constant factor in (21) is not the best possible.

For $p>1$, we find $\widehat{\Psi}^{1-p}(n)=\frac{v_{n}}{\left(V_{n}-\tau_{n}\right)^{1-p \sigma}}(n \in \mathbf{N}), \Phi_{\delta}^{1-q}(x)=\frac{\mu(x)}{U^{1-q \delta \sigma}(x)}\left(x \in \mathbf{R}_{+}\right)$, and we define the following real normed spaces:

$$
\begin{aligned}
& L_{p, \Phi_{\delta}}\left(\mathbf{R}_{+}\right)=\left\{f ; f=f(x), x \in \mathbf{R}_{+},\|f\|_{p, \Phi_{\delta}}<\infty\right\}, \\
& l_{q, \widehat{\Psi}}=\left\{a ; a=\left\{a_{n}\right\}_{n=1}^{\infty},\|a\|_{q, \widehat{\Psi}}<\infty\right\}, \\
& L_{q, \Phi_{\delta}^{1-q}}\left(\mathbf{R}_{+}\right)=\left\{h ; h=h(x), x \in \mathbf{R}_{+},\|h\|_{\left.q, \Phi_{\delta}^{1-q}<\infty\right\},},\right. \\
& l_{p, \widehat{\Psi}^{1-p}}=\left\{c ; c=\left\{c_{n}\right\}_{n=1}^{\infty},\|c\|_{p, \widehat{\Psi}^{1-p}}<\infty\right\} .
\end{aligned}
$$

Assuming that $f \in L_{p, \Phi_{\delta}}\left(\mathbf{R}_{+}\right)$, setting

$$
c=\left\{c_{n}\right\}_{n=1}^{\infty}, \quad c_{n}:=\int_{0}^{\infty} \frac{1}{e^{\alpha U^{\delta \gamma}(x)\left(V_{n}-\tau_{n}\right)^{\gamma}}} f(x) d x, \quad n \in \mathbf{N},
$$

we can rewrite (22) as $\|c\|_{p, \widehat{\Psi} 1-p}<k(\sigma)\|f\|_{p, \Phi_{\delta}}<\infty$, namely, $c \in l_{p, \widehat{\Psi}^{1-p}}$.

Definition 1 Define a half-discrete Hardy-Hilbert-type operator $T_{1}: L_{p, \Phi_{\delta}}\left(\mathbf{R}_{+}\right) \rightarrow l_{p, \widehat{\Psi} 1-p}$ as follows: For any $f \in L_{p, \Phi_{\delta}}\left(\mathbf{R}_{+}\right)$, there exists a unique representation $T_{1} f=c \in l_{p, \widehat{\Psi} 1-p}$. Define the formal inner product of $T_{1} f$ and $a=\left\{a_{n}\right\}_{n=1}^{\infty} \in l_{q, \widehat{\Psi}}$ as follows:

$$
\left(T_{1} f, a\right):=\sum_{n=1}^{\infty}\left[\int_{0}^{\infty} \frac{1}{e^{\alpha U^{\delta \gamma}(x)\left(V_{n}-\tau_{n}\right)} f} f(x) d x\right] a_{n} .
$$

Then we can rewrite (21) and (22) as follows:

$$
\begin{aligned}
& \left(T_{1} f, a\right)<k(\sigma)\|f\|_{p, \Phi_{\delta}}\|a\|_{q, \widehat{\Psi}}, \\
& \left\|T_{1} f\right\|_{p, \widehat{\Psi} 1-p}<k(\sigma)\|f\|_{p, \Phi_{\delta}} .
\end{aligned}
$$


Define the norm of operator $T_{1}$ as follows:

$$
\left\|T_{1}\right\|:=\sup _{f(\neq \theta) \in L_{p, \Phi_{\delta}}\left(\mathbf{R}_{+}\right)} \frac{\left\|T_{1} f\right\|_{p, \widehat{\Psi}^{1-p}}}{\|f\|_{p, \Phi_{\delta}}}
$$

Then by (36), it follows that $\left\|T_{1}\right\| \leq k(\sigma)$. Since, by Theorem 2 , the constant factor in (36) is the best possible, we have

$$
\left\|T_{1}\right\|=k(\sigma)=\frac{\Gamma(\sigma / \gamma)}{\gamma \alpha^{\sigma / \gamma}} .
$$

Assuming that $a=\left\{a_{n}\right\}_{n=1}^{\infty} \in l_{q, \widehat{\Psi}}$, setting

$$
h(x):=\sum_{n=1}^{\infty} \frac{1}{e^{\alpha U^{\delta \gamma}(x)\left(V_{n}-\tau_{n}\right)^{\gamma}}} a_{n}, \quad x \in \mathbf{R}_{+},
$$

we can rewrite (23) as $\|h\|_{q, \Phi_{\delta}^{1-q}}<k(\sigma)\|a\|_{q, \widehat{\Psi}}<\infty$, namely, $h \in L_{q, \Phi_{\delta}^{1-q}}\left(\mathbf{R}_{+}\right)$.

Definition 2 Define a half-discrete Hardy-Hilbert-type operator $T_{2}: l_{q, \widehat{\Psi}} \rightarrow L_{q, \Phi_{\delta}^{1-q}}\left(\mathbf{R}_{+}\right)$ as follows: For any $a=\left\{a_{n}\right\}_{n=1}^{\infty} \in l_{q, \widehat{\Psi}}$, there exists a unique representation $T_{2} a=h \in$ $L_{q, \Phi_{\delta}^{1-q}}\left(\mathbf{R}_{+}\right)$. Define the formal inner product of $T_{2} a$ and $f \in L_{p, \Phi_{\delta}}\left(\mathbf{R}_{+}\right)$as follows:

$$
\left(T_{2} a, f\right):=\int_{0}^{\infty}\left[\sum_{n=1}^{\infty} \frac{1}{e^{\alpha L^{\delta \gamma}(x)\left(V_{n}-\tau_{n}\right)^{\gamma}}} a_{n}\right] f(x) d x .
$$

Then we can rewrite (21) and (23) as follows:

$$
\begin{aligned}
& \left(T_{2} a, f\right)<k(\sigma)\|f\|_{p, \Phi_{\delta}}\|a\|_{q, \widehat{\Psi}}, \\
& \left\|T_{2} a\right\|_{q, \Phi_{\delta}^{1-q}}<k(\sigma)\|a\|_{q, \widehat{\Psi}} .
\end{aligned}
$$

Define the norm of operator $T_{2}$ as follows:

$$
\left\|T_{2}\right\|:=\sup _{a(\neq \theta) \in l_{q, \widehat{\Psi}}} \frac{\left\|T_{2} a\right\|_{q, \Phi_{\delta}^{1-q}}}{\|a\|_{q, \widehat{\Psi}}} .
$$

Then by (40), we find $\left\|T_{2}\right\| \leq k(\sigma)$. Since, by Theorem 2 , the constant factor in (40) is the best possible, we have

$$
\left\|T_{2}\right\|=k(\sigma)=\frac{\Gamma(\sigma / \gamma)}{\gamma \alpha^{\sigma / \gamma}}=\left\|T_{1}\right\|
$$

\section{Some equivalent reverses}

In the following, we also set

$$
\widetilde{\Phi}_{\delta}(x):=\left(1-\theta_{\delta}(\sigma, x)\right) \frac{U^{p(1-\delta \sigma)-1}(x)}{\mu^{p-1}(x)} \quad\left(x \in \mathbf{R}_{+}\right) .
$$

For $0<p<1$ or $p<0$, we still use the formal symbols $\|f\|_{p, \Phi_{\delta}},\|f\|_{p, \widetilde{\Phi}_{\delta}}$, and $\|a\|_{q, \widehat{\Psi}}$. 
Theorem 3 As regards the assumptions of Theorem 2, for $p<0,0<\|f\|_{p, \Phi_{\delta}},\|a\|_{q, \widehat{\Psi}}<\infty$, we have the following equivalent inequalities with the best possible constant factor $k(\sigma)=$ $\frac{\Gamma(\sigma / \gamma)}{\gamma \alpha^{\sigma / \gamma}}$ :

$$
\begin{aligned}
& I=\sum_{n=1}^{\infty} \int_{0}^{\infty} \frac{a_{n} f(x)}{e^{\alpha U^{\delta \gamma}(x)\left(V_{n}-\tau_{n}\right)^{\gamma}}} d x>\frac{\Gamma(\sigma / \gamma)}{\gamma \alpha^{\sigma / \gamma}}\|f\|_{p, \Phi_{\delta}}\|a\|_{q, \widehat{\Psi}}, \\
& J_{1}=\sum_{n=1}^{\infty} \frac{v_{n}}{\left(V_{n}-\tau_{n}\right)^{1-p \sigma}}\left[\int_{0}^{\infty} \frac{f(x)}{e^{\alpha U^{\delta \gamma}(x)\left(V_{n}-\tau_{n}\right)^{\gamma}}} d x\right]^{p}>\frac{\Gamma(\sigma / \gamma)}{\gamma \alpha^{\sigma / \gamma}}\|f\|_{p, \Phi_{\delta}}, \\
& J_{2}=\left\{\int_{0}^{\infty} \frac{\mu(x)}{U^{1-q \delta \sigma}(x)}\left[\sum_{n=1}^{\infty} \frac{a_{n}}{e^{\alpha U^{\delta \gamma}(x)\left(V_{n}-\tau_{n}\right)^{\gamma}}}\right]^{q} d x\right\}^{\frac{1}{q}}>\frac{\Gamma(\sigma / \gamma)}{\gamma \alpha^{\sigma / \gamma}}\|a\|_{q, \widehat{\Psi}} .
\end{aligned}
$$

Proof By the reverse Hölder inequality with weight (cf. [41]), since $p<0$, in the similar way to obtaining (24) and (25), we have

$$
\begin{aligned}
& {\left[\int_{0}^{\infty} \frac{1}{e^{\alpha U^{\delta \gamma}(x)\left(V_{n}-\tau_{n}\right)^{\gamma}}} f(x) d x\right]^{p}} \\
& \quad \leq \frac{\left(\varpi_{\delta}(\sigma, n)\right)^{p-1}}{\left(V_{n}-\tau_{n}\right)^{p \sigma-1} v_{n}} \int_{0}^{\infty} \frac{1}{e^{\alpha U^{\delta \gamma}(x)\left(V_{n}-\tau_{n}\right)^{\gamma}}} \frac{U^{(1-\delta \sigma)(p-1)}(x) v_{n}}{\left(V_{n}-\tau_{n}\right)^{1-\sigma} \mu^{p-1}(x)} f^{p}(x) d x .
\end{aligned}
$$

Then by (18) and the Lebesgue term by term integration theorem, it follows that

$$
\begin{aligned}
J_{1} & \geq(k(\sigma))^{\frac{1}{q}}\left[\sum_{n=1}^{\infty} \int_{0}^{\infty} \frac{1}{e^{\alpha U^{\delta \gamma}(x)\left(V_{n}-\tau_{n}\right)^{\gamma}}} \frac{U^{(1-\delta \sigma)(p-1)}(x) v_{n}}{\left(V_{n}-\tau_{n}\right)^{1-\sigma} \mu^{p-1}(x)} f^{p}(x) d x\right]^{\frac{1}{p}} \\
& =(k(\sigma))^{\frac{1}{q}}\left[\int_{0}^{\infty} \omega_{\delta}(\sigma, x) \frac{U^{p(1-\delta \sigma)-1}(x)}{\mu^{p-1}(x)} f^{p}(x) d x\right]^{\frac{1}{p}} .
\end{aligned}
$$

Then by (16), we have (43).

By the reverse Hölder inequality ( $c f$. [41]), we have

$$
\begin{aligned}
I & =\sum_{n=1}^{\infty}\left[\frac{v_{n}^{\frac{1}{p}}}{\left(V_{n}-\tau_{n}\right)^{\frac{1}{p}-\sigma}} \int_{0}^{\infty} \frac{f(x)}{e^{\alpha U^{\delta \gamma}(x)\left(V_{n}-\tau_{n}\right)^{\gamma}}} d x\right]\left[\frac{\left(V_{n}-\tau_{n}\right)^{\frac{1}{p}-\sigma} a_{n}}{v_{n}^{1 / p}}\right] \\
& \geq J_{1}\|a\|_{q, \widehat{\Psi}} .
\end{aligned}
$$

Then by (43), we have (42). On the other hand, assuming that (42) is valid, we set $a_{n}$ as in Theorem 1 . Then we find $J_{1}^{p}=\|a\|_{q, \widehat{\Psi}}^{q}$. If $J_{1}=\infty$, then (43) is trivially valid; if $J_{1}=0$, then (43) keeps impossible. Suppose that $0<J_{1}<\infty$. By (42), it follows that

$$
\|a\|_{q, \widehat{\Psi}}^{q}=J_{1}^{p}=I>k(\sigma)\|f\|_{p, \Phi_{\delta}}\|a\|_{q, \widehat{\Psi}}, \quad\|a\|_{q, \widehat{\Psi}}^{q-1}=J_{1}>k(\sigma)\|f\|_{p, \Phi_{\delta}},
$$

and then (43) follows, which is equivalent to (42). 
Still by the reverse Hölder's inequality with weight (cf. [41]), since $0<q<1$, in the similar way to obtaining (27) and (28), we have

$$
\begin{aligned}
& {\left[\sum_{n=1}^{\infty} \frac{1}{e^{\alpha U^{\delta \gamma}(x)\left(V_{n}-\tau_{n}\right)^{\gamma}}} a_{n}\right]^{q}} \\
& \quad \geq \frac{\left(\omega_{\delta}(\sigma, x)\right)^{q-1}}{U^{q \delta \sigma-1}(x) \mu(x)} \sum_{n=1}^{\infty} \frac{1}{e^{\alpha U^{\delta \gamma}(x)\left(V_{n}-\tau_{n}\right)^{\gamma}}} \frac{\left(V_{n}-\tau_{n}\right)^{(1-\sigma)(q-1)} \mu(x)}{U^{1-\delta \sigma}(x) v_{n}^{q-1}} a_{n}^{q} .
\end{aligned}
$$

Then by (16) and the Lebesgue term by term integration theorem, it follows that

$$
\begin{aligned}
& J_{2}>(k(\sigma))^{\frac{1}{p}}\left[\int_{0}^{\infty} \sum_{n=1}^{\infty} \frac{1}{e^{\alpha U^{\delta \gamma}(x)\left(V_{n}-\tau_{n}\right)^{\gamma}}} \frac{\left(V_{n}-\tau_{n}\right)^{(1-\sigma)(q-1)} \mu(x)}{U^{1-\delta \sigma}(x) \nu_{n}^{q-1}} a_{n}^{q} d x\right]^{\frac{1}{q}} \\
& =(k(\sigma))^{\frac{1}{p}}\left[\sum_{n=1}^{\infty} \varpi_{\delta}(\sigma, n) \frac{\left(V_{n}-\tau_{n}\right)^{q(1-\sigma)-1}}{v_{n}^{q-1}} a_{n}^{q}\right]^{\frac{1}{q}} .
\end{aligned}
$$

Then by (18), we have (44).

By the reverse Hölder inequality ( $c f$. [41]), we have

$$
\begin{aligned}
I & =\int_{0}^{\infty}\left(\frac{U^{\frac{1}{q}-\delta \sigma}(x)}{\mu^{\frac{1}{q}}(x)} f(x)\right)\left[\frac{\mu^{\frac{1}{q}}(x)}{U^{\frac{1}{q}-\delta \sigma}(x)} \sum_{n=1}^{\infty} \frac{a_{n}}{e^{\alpha U^{\delta \gamma}(x)\left(V_{n}-\tau_{n}\right)^{\gamma}}}\right] d x \\
& \geq\|f\|_{p, \Phi_{\delta}} J_{2} .
\end{aligned}
$$

Then by (44), we have (42). On the other hand, assuming that (44) is valid, we set $f(x)$ as in Theorem 1 . Then we find $J_{2}^{q}=\|f\|_{p, \Phi_{\delta}}^{p}$. If $J_{2}=\infty$, then (44) is trivially valid; if $J_{2}=0$, then (44) remains impossible. Suppose that $0<J_{2}<\infty$. By (42), it follows that

$$
\|f\|_{p, \Phi_{\delta}}^{p}=J_{2}^{q}=I>k(\sigma)\|f\|_{p, \Phi_{\delta}}\|a\|_{q, \widehat{\Psi}}, \quad\|f\|_{p, \Phi_{\delta}}^{p-1}=J_{2}>k(\sigma)\|a\|_{q, \widehat{\Psi}},
$$

and then (44) follows, which is equivalent to (42).

Therefore, inequalities (42), (43), and (44) are equivalent.

For $\varepsilon \in(0, q \sigma)$, we set $\widetilde{\sigma}=\sigma-\frac{\varepsilon}{q}(\in(0,1))$ and $\tilde{f}=\widetilde{f}(x), x \in \mathbf{R}_{+}, \widetilde{a}=\left\{\widetilde{a}_{n}\right\}_{n=1}^{\infty}$,

$$
\begin{aligned}
& \tilde{f}(x)= \begin{cases}U^{\delta(\widetilde{\sigma}+\varepsilon)-1}(x) \mu(x), & 0<x^{\delta} \leq 1, \\
0, & x^{\delta}>0,\end{cases} \\
& \tilde{a}_{n}=\left(V_{n}-\tau_{n}\right)^{\widetilde{\sigma}-1} v_{n}=\left(V_{n}-\tau_{n}\right)^{\sigma-\frac{\varepsilon}{q}-1} v_{n}, \quad n \in \mathbf{N} .
\end{aligned}
$$

By (20), (32), and (16), we obtain

$$
\begin{aligned}
& \|\widetilde{f}\|_{p, \Phi_{\delta}}\|\widetilde{a}\|_{q, \widehat{\Psi}}=\frac{1}{\varepsilon} U^{\frac{\delta \varepsilon}{p}}(1)\left(\frac{1}{v_{1}^{\varepsilon}}+\varepsilon O(1)\right)^{\frac{1}{q}}, \\
& \widetilde{I}=\sum_{n=1}^{\infty} \int_{0}^{\infty} \frac{\widetilde{a}_{n} \widetilde{f}(x)}{e^{\alpha U^{\delta \gamma}(x)\left(V_{n}-\tau_{n}\right)^{\gamma}}} d x=\int_{\left\{x>0 ; 0<x^{\delta} \leq 1\right\}} \omega_{\delta}(\widetilde{\sigma}, x) \frac{\mu(x)}{U^{1-\delta \varepsilon}(x)} d x \\
& \quad \leq k(\widetilde{\sigma}) \int_{\left\{x>0 ; 0<x^{\delta} \leq 1\right\}} \frac{\mu(x)}{U^{1-\delta \varepsilon}(x)} d x=\frac{1}{\varepsilon} k\left(\sigma-\frac{\varepsilon}{q}\right) U^{\delta \varepsilon}(1) .
\end{aligned}
$$


If there exists a positive constant $K \geq k(\sigma)$, such that (42) is valid when replacing $k(\sigma)$ to $K$, then in particular, we have $\varepsilon \widetilde{I}>\varepsilon K\|\widetilde{f}\|_{p, \Phi_{\delta}}\|\widetilde{a}\|_{q, \widehat{\Psi}}$, namely,

$$
k\left(\sigma-\frac{\varepsilon}{q}\right) U^{\delta \varepsilon}(1)>K \cdot U^{\frac{\delta \varepsilon}{p}}(1)\left(\frac{1}{v_{1}^{\varepsilon}}+\varepsilon O(1)\right)^{\frac{1}{q}}
$$

It follows that $k(\sigma) \geq K\left(\varepsilon \rightarrow 0^{+}\right)$. Hence, $K=k(\sigma)$ is the best possible constant factor of (42).

The constant factor $k(\sigma)$ in (43) ((44)) is still the best possible. Otherwise, we would reach a contradiction by $(45)((46))$ that the constant factor in (42) is not the best possible.

Theorem 4 As regards the assumptions of Theorem 2 , if $0<p<1,0<\|f\|_{p, \Phi_{\delta}},\|a\|_{q, \widehat{\Psi}}<\infty$, then we have the following equivalent inequalities with the best possible constant factor $k(\sigma)=\frac{\Gamma(\sigma / \gamma)}{\gamma \alpha^{\sigma / \gamma}}:$

$$
\begin{aligned}
& I=\sum_{n=1}^{\infty} \int_{0}^{\infty} \frac{a_{n} f(x)}{e^{\alpha U^{\delta \gamma}(x)\left(V_{n}-\tau_{n}\right)^{\gamma}}} d x>\frac{\Gamma(\sigma / \gamma)}{\gamma \alpha^{\sigma / \gamma}}\|f\|_{p, \widetilde{\Phi}_{\delta}}\|a\|_{q, \widehat{\Psi}}, \\
& J_{1}=\sum_{n=1}^{\infty} \frac{v_{n}}{\left(V_{n}-\tau_{n}\right)^{1-p \sigma}}\left[\int_{0}^{\infty} \frac{f(x) d x}{e^{\alpha L^{\delta \gamma}(x)\left(V_{n}-\tau_{n}\right)^{\gamma}}}\right]^{p}>\frac{\Gamma(\sigma / \gamma)}{\gamma \alpha^{\sigma / \gamma}}\|f\|_{p, \widetilde{\Phi}_{\delta}}, \\
& J:=\left\{\int_{0}^{\infty} \frac{\left(1-\theta_{\delta}(\sigma, x)\right)^{1-q} \mu(x)}{U^{1-q \delta \sigma}(x)}\left[\sum_{n=1}^{\infty} \frac{a_{n}}{e^{\alpha U^{\delta \gamma}(x)\left(V_{n}-\tau_{n}\right)^{\gamma}}}\right]^{q} d x\right\}^{\frac{1}{q}} \\
& >\frac{\Gamma(\sigma / \gamma)}{\gamma \alpha^{\sigma / \gamma}}\|a\|_{q, \widehat{\Psi}} .
\end{aligned}
$$

Proof By the reverse Hölder inequality with weight (cf. [41]), since $0<p<1$, in a similar way to obtaining (24) and (25), we have

$$
\begin{aligned}
& {\left[\int_{0}^{\infty} \frac{f(x)}{e^{\alpha U^{\delta \gamma}(x)\left(V_{n}-\tau_{n}\right)^{\gamma}}} d x\right]^{p}} \\
& \quad \geq \frac{\left(\varpi_{\delta}(\sigma, n)\right)^{p-1}}{\left(V_{n}-\tau_{n}\right)^{p \sigma-1} v_{n}} \int_{0}^{\infty} \frac{1}{e^{\alpha U^{\delta \gamma}(x)\left(V_{n}-\tau_{n}\right)^{\gamma}}} \frac{U^{(1-\delta \sigma)(p-1)}(x) v_{n}}{\left(V_{n}-\tau_{n}\right)^{1-\sigma} \mu^{p-1}(x)} f^{p}(x) d x .
\end{aligned}
$$

In view of (18) and the Lebesgue term by term integration theorem, we find

$$
\begin{aligned}
J_{1} & \geq(k(\sigma))^{\frac{1}{q}}\left[\sum_{n=1}^{\infty} \int_{0}^{\infty} \frac{1}{e^{\alpha U^{\delta \gamma}(x)\left(V_{n}-\tau_{n}\right)^{\gamma}}} \frac{U^{(1-\delta \sigma)(p-1)}(x) v_{n}}{\left(V_{n}-\tau_{n}\right)^{1-\sigma} \mu^{p-1}(x)} f^{p}(x) d x\right]^{\frac{1}{p}} \\
& =(k(\sigma))^{\frac{1}{q}}\left[\int_{0}^{\infty} \omega_{\delta}(\sigma, x) \frac{U^{p(1-\delta \sigma)-1}(x)}{\mu^{p-1}(x)} f^{p}(x) d x\right]^{\frac{1}{p}} .
\end{aligned}
$$

Then by (19), we have (48). 
By the reverse Hölder inequality (cf. [41]), we have

$$
\begin{aligned}
I & =\sum_{n=1}^{\infty}\left[\frac{v_{n}^{1 / p}}{\left(V_{n}-\tau_{n}\right)^{\frac{1}{p}-\sigma}} \int_{0}^{\infty} \frac{f(x)}{e^{\alpha U^{\delta \gamma}(x)\left(V_{n}-\tau_{n}\right)^{\gamma}}} d x\right]\left[\frac{\left(V_{n}-\tau_{n}\right)^{\frac{1}{p}-\sigma} a_{n}}{v_{n}^{1 / p}}\right] \\
& \geq J_{1}\|a\|_{q, \widehat{\Psi}} .
\end{aligned}
$$

Then by (48), we have (47). On the other hand, assuming that (47) is valid, we set $a_{n}$ as in Theorem 1. Then we find $J_{1}^{p}=\|a\|_{q, \widehat{\Psi}}^{q}$. If $J_{1}=\infty$, then (48) is trivially valid; if $J_{1}=0$, then (48) remains impossible. Suppose that $0<J_{1}<\infty$. By (47), it follows that

$$
\|a\|_{q, \widehat{\Psi}}^{q}=J_{1}^{p}=I>k(\sigma)\|f\|_{p, \widetilde{\Phi}_{\delta}}\|a\|_{q, \widehat{\Psi}}, \quad\|a\|_{q, \widehat{\Psi}}^{q-1}=J_{1}>k(\sigma)\|f\|_{p, \widetilde{\Phi}_{\delta}},
$$

and then (48) follows, which is equivalent to (47).

Still by the reverse Hölder inequality with weight ( $c f$. [41]), since $q<0$, we have

$$
\begin{aligned}
& {\left[\sum_{n=1}^{\infty} \frac{a_{n}}{e^{\alpha U^{\delta \gamma}(x)\left(V_{n}-\tau_{n}\right)^{\gamma}}}\right]^{q}} \\
& \quad \leq \frac{\left(\omega_{\delta}(\sigma, x)\right)^{q-1}}{U^{q \delta \sigma-1}(x) \mu(x)} \sum_{n=1}^{\infty} \frac{1}{e^{\alpha U^{\delta \gamma}(x)\left(V_{n}-\tau_{n}\right)^{\gamma}}} \frac{\left(V_{n}-\tau_{n}\right)^{(1-\sigma)(q-1)} \mu(x)}{U^{1-\delta \sigma}(x) v_{n}^{q-1}} a_{n}^{q} .
\end{aligned}
$$

Then by (19) and the Lebesgue term by term integration theorem, it follows that

$$
\begin{aligned}
J & >(k(\sigma))^{\frac{1}{p}}\left[\int_{0}^{\infty} \sum_{n=1}^{\infty} \frac{1}{e^{\alpha U^{\delta \gamma}(x)\left(V_{n}-\tau_{n}\right)^{\gamma}}} \frac{\left(V_{n}-\tau_{n}\right)^{(1-\sigma)(q-1)} \mu(x)}{U^{1-\delta \sigma}(x) v_{n}^{q-1}} a_{n}^{q} d x\right]^{\frac{1}{q}} \\
& =(k(\sigma))^{\frac{1}{p}}\left[\sum_{n=1}^{\infty} \varpi_{\delta}(\sigma, n) \frac{\left(V_{n}-\tau_{n}\right)^{q(1-\sigma)-1}}{v_{n}^{q-1}} a_{n}^{q}\right]^{\frac{1}{q}} .
\end{aligned}
$$

Then by (18), we have (49).

By the reverse Hölder inequality ( $c f$. [41]), we have

$$
\begin{aligned}
I= & \int_{0}^{\infty}\left[\left(1-\theta_{\delta}(\sigma, x)\right)^{\frac{1}{p}} \frac{U^{\frac{1}{q}-\delta \sigma}(x)}{\mu^{\frac{1}{q}}(x)} f(x)\right] \\
& \times\left[\frac{\left(1-\theta_{\delta}(\sigma, x)\right)^{\frac{-1}{p}} \mu^{\frac{1}{q}}(x)}{U^{\frac{1}{q}-\delta \sigma}(x)} \sum_{n=1}^{\infty} \frac{a_{n}}{e^{\alpha U^{\delta \gamma}(x)\left(V_{n}-\tau_{n}\right)^{\gamma}}}\right] d x \\
\geq & \|f\|_{p, \widetilde{\Phi}_{\delta} J .}
\end{aligned}
$$

Then by (49), we have (47). On the other hand, assuming that (47) is valid, we set $f(x)$ as in Theorem 1 . Then we find $J^{q}=\|f\|_{p, \widetilde{\Phi}_{\delta}}^{p}$. If $J=\infty$, then (49) is trivially valid; if $J=0$, then (49) keeps impossible. Suppose that $0<J<\infty$. By (47), it follows that

$$
\|f\|_{p, \widetilde{\Phi}_{\delta}}^{p}=J^{q}=I>k(\sigma)\|f\|_{p, \widetilde{\Phi}_{\delta}}\|a\|_{q, \widehat{\Psi}}, \quad\|f\|_{p, \widetilde{\Phi}_{\delta}}^{p-1}=J>k(\sigma)\|a\|_{q, \widehat{\Psi}}
$$

and then (49) follows, which is equivalent to (47). 
Therefore, inequalities (47), (48), and (49) are equivalent.

$$
\begin{aligned}
& \text { For } \varepsilon \in(0, p \sigma) \text {, we set } \widetilde{\sigma}=\sigma+\frac{\varepsilon}{p} \text { and } \widetilde{f}=\widetilde{f}(x), x \in \mathbf{R}_{+}, \widetilde{a}=\left\{\widetilde{a}_{n}\right\}_{n=1}^{\infty}, \\
& \tilde{f}(x)= \begin{cases}U^{\delta \widetilde{\sigma}-1}(x) \mu(x), & 0<x^{\delta} \leq 1, \\
0, & x^{\delta}>0,\end{cases} \\
& \tilde{a}_{n}=\left(V_{n}-\tau_{n}\right)^{\widetilde{\sigma}-\varepsilon-1} v_{n}=\left(V_{n}-\tau_{n}\right)^{\sigma-\frac{\varepsilon}{q}-1} v_{n}, \quad n \in \mathbf{N} .
\end{aligned}
$$

By (19), (20), and (32), we obtain

$$
\begin{aligned}
& \|\widetilde{f}\|_{p, \widetilde{\Phi}_{\delta}}\|\widetilde{a}\|_{q, \widehat{\Psi}} \\
& =\left[\int_{\left\{x>0 ; 0<x^{\delta} \leq 1\right\}}\left(1-O\left((U(x))^{\delta \sigma}\right)\right) \frac{\mu(x) d x}{U^{1-\delta \varepsilon}(x)}\right]^{\frac{1}{p}}\left[\sum_{n=1}^{\infty} \frac{v_{n}}{\left(V_{n}-\tau_{n}\right)^{1+\varepsilon}}\right]^{\frac{1}{q}} \\
& =\frac{1}{\varepsilon}\left(U^{\delta \varepsilon}(1)-\varepsilon O_{1}(1)\right)^{\frac{1}{p}}\left(\frac{1}{v_{1}^{\varepsilon}}+\varepsilon O(1)\right)^{\frac{1}{q}}, \\
& \widetilde{I}=\sum_{n=1}^{\infty} \int_{0}^{\infty} \frac{1}{e^{\alpha U^{\delta \gamma}(x)\left(V_{n}-\tau_{n}\right)^{\gamma}}} \widetilde{a}_{n} \tilde{f}(x) d x \\
& =\sum_{n=1}^{\infty}\left[\int_{\left\{x>0 ; 0<x^{\delta} \leq 1\right\}} \frac{1}{e^{\alpha U^{\delta \gamma}(x)\left(V_{n}-\tau_{n}\right)^{\gamma}}} \frac{\left(V_{n}-\tau_{n}\right)^{\tilde{\sigma}} \mu(x)}{U^{1-\delta \widetilde{\sigma}}(x)} d x\right] \frac{v_{n}}{\left(V_{n}-\tau_{n}\right)^{1+\varepsilon}} \\
& \leq \sum_{n=1}^{\infty}\left[\int_{0}^{\infty} \frac{1}{e^{\alpha U^{\delta \gamma}(x)\left(V_{n}-\tau_{n}\right)^{\gamma}}} \frac{\left(V_{n}-\tau_{n}\right)^{\tilde{\sigma}} \mu(x)}{U^{1-\delta \widetilde{\sigma}}(x)} d x\right] \frac{v_{n}}{\left(V_{n}-\beta\right)^{1+\varepsilon}} \\
& =\sum_{n=1}^{\infty} \varpi_{\delta}(\widetilde{\sigma}, n) \frac{v_{n}}{\left(V_{n}-\tau_{n}\right)^{1+\varepsilon}}=k(\widetilde{\sigma}) \sum_{n=1}^{\infty} \frac{v_{n}}{\left(V_{n}-\tau_{n}\right)^{1+\varepsilon}} \\
& =\frac{1}{\varepsilon} k\left(\sigma+\frac{\varepsilon}{p}\right)\left(\frac{1}{v_{1}^{\varepsilon}}+\varepsilon O(1)\right) \text {. }
\end{aligned}
$$

If there exists a positive constant $K \geq k(\sigma)$, such that (42) is valid when replacing $k(\sigma)$ to $K$, then, in particular, we have $\varepsilon \widetilde{I}>\varepsilon K\|\widetilde{f}\|_{p, \widetilde{\Phi_{\delta}}}\|\widetilde{a}\|_{q, \widehat{\Psi}}$, namely,

$$
\begin{aligned}
& k\left(\sigma+\frac{\varepsilon}{p}\right)\left(\frac{1}{v_{1}^{\varepsilon}}+\varepsilon O(1)\right) \\
& \quad>K\left(U^{\delta \varepsilon}(1)-\varepsilon O_{1}(1)\right)^{\frac{1}{p}}\left(\frac{1}{v_{1}^{\varepsilon}}+\varepsilon O(1)\right)^{\frac{1}{q}} .
\end{aligned}
$$

It follows that $k(\sigma) \geq K\left(\varepsilon \rightarrow 0^{+}\right)$. Hence, $K=k(\sigma)$ is the best possible constant factor of (47).

The constant factor $k(\sigma)$ in (48) ((49)) is still the best possible. Otherwise, we would reach the contradiction by $(50)((51))$ that the constant factor in $(47)$ is not the best possible.

\section{Some corollaries and a remark}

For $\delta=1$ in Theorems 2-4, we have the following inequalities with the non-homogeneous kernel. 
Corollary 1 As regards the assumptions of Theorem 2, (i) for $p>1,0<\|f\|_{p, \Phi_{1}},\|a\|_{q, \widehat{\Psi}}<\infty$, we have the following equivalent inequalities:

$$
\begin{aligned}
& \sum_{n=1}^{\infty} \int_{0}^{\infty} \frac{a_{n} f(x)}{e^{\alpha U^{\delta \gamma}(x)\left(V_{n}-\tau_{n}\right)^{\gamma}}} d x<\frac{\Gamma(\sigma / \gamma)}{\gamma \alpha^{\sigma / \gamma}}\|f\|_{p, \Phi_{1}}\|a\|_{q, \widehat{\Psi}}, \\
& \sum_{n=1}^{\infty} \frac{v_{n}}{\left(V_{n}-\tau_{n}\right)^{1-p \sigma}}\left[\int_{0}^{\infty} \frac{f(x)}{e^{\alpha U^{\gamma}(x)\left(V_{n}-\tau_{n}\right)^{\gamma}}} d x\right]^{p}<\frac{\Gamma(\sigma / \gamma)}{\gamma \alpha^{\sigma / \gamma}}\|f\|_{p, \Phi_{1}}, \\
& \left\{\int_{0}^{\infty} \frac{\mu(x)}{U^{1-q \sigma}(x)}\left[\sum_{n=1}^{\infty} \frac{a_{n}}{e^{\alpha U^{\gamma}(x)\left(V_{n}-\tau_{n}\right)^{\gamma}}}\right]^{q} d x\right\}^{\frac{1}{q}}<\frac{\Gamma(\sigma / \gamma)}{\gamma \alpha^{\sigma / \gamma}}\|a\|_{q, \widehat{\Psi}} ;
\end{aligned}
$$

(ii) for $p<0,0<\|f\|_{p, \Phi_{1}},\|a\|_{q, \widehat{\Psi}}<\infty$, we have the following equivalent inequalities:

$$
\begin{aligned}
& \sum_{n=1}^{\infty} \int_{0}^{\infty} \frac{a_{n} f(x)}{e^{\alpha U^{\gamma}(x)\left(V_{n}-\tau_{n}\right)^{\gamma}}} d x>\frac{\Gamma(\sigma / \gamma)}{\gamma \alpha^{\sigma / \gamma}}\|f\|_{p, \Phi_{1}}\|a\|_{q, \widehat{\Psi}}, \\
& \sum_{n=1}^{\infty} \frac{v_{n}}{\left(V_{n}-\tau_{n}\right)^{1-p \sigma}}\left[\int_{0}^{\infty} \frac{f(x)}{e^{\alpha U^{\gamma}(x)\left(V_{n}-\tau_{n}\right)^{\gamma}}} d x\right]^{p}>\frac{\Gamma(\sigma / \gamma)}{\gamma \alpha^{\sigma / \gamma}}\|f\|_{p, \Phi_{1}}, \\
& \left\{\int_{0}^{\infty} \frac{\mu(x)}{U^{1-q \sigma}(x)}\left[\sum_{n=1}^{\infty} \frac{a_{n}}{e^{\alpha U^{\gamma}(x)\left(V_{n}-\tau_{n}\right)^{\gamma}}}\right]^{q} d x\right\}^{\frac{1}{q}}>\frac{\Gamma(\sigma / \gamma)}{\gamma \alpha^{\sigma / \gamma}}\|a\|_{q, \widehat{\Psi}} ;
\end{aligned}
$$

(iii) for $0<p<1,0<\|f\|_{p, \Phi_{1}},\|a\|_{q, \widehat{\Psi}}<\infty$, we have the following equivalent inequalities:

$$
\begin{aligned}
& \sum_{n=1}^{\infty} \int_{0}^{\infty} \frac{a_{n} f(x)}{e^{\alpha U^{\gamma}(x)\left(V_{n}-\tau_{n}\right)^{\gamma}}} d x>\frac{\Gamma(\sigma / \gamma)}{\gamma \alpha^{\sigma / \gamma}}\|f\|_{p, \widetilde{\Phi}_{1}}\|a\|_{q, \widehat{\Psi}}, \\
& \sum_{n=1}^{\infty} \frac{v_{n}}{\left(V_{n}-\tau_{n}\right)^{1-p \sigma}}\left[\int_{0}^{\infty} \frac{f(x)}{e^{\alpha U^{\gamma}(x)\left(V_{n}-\tau_{n}\right)^{\gamma}}} d x\right]^{p}>\frac{\Gamma(\sigma / \gamma)}{\gamma \alpha^{\sigma / \gamma}}\|f\|_{p, \widetilde{\Phi}_{1},}, \\
& \left\{\int_{0}^{\infty} \frac{\left(1-\theta_{1}(\sigma, x)\right)^{1-q} \mu(x)}{U^{1-q \sigma}(x)}\left[\sum_{n=1}^{\infty} \frac{a_{n}}{e^{\alpha U^{\gamma}(x)\left(V_{n}-\tau_{n}\right)^{\gamma}}}\right]^{q} d x\right\}^{\frac{1}{q}} \\
& >\frac{\Gamma(\sigma / \gamma)}{\gamma \alpha^{\sigma / \gamma}}\|a\|_{q, \widehat{\Psi}} .
\end{aligned}
$$

The above inequalities are with the best possible constant factor $\frac{\Gamma(\sigma / \gamma)}{\gamma \alpha^{\sigma / \gamma}}$.

For $\delta=-1$ in Theorems 2-4, we have the following inequalities with the homogeneous kernel of degree 0 :

Corollary 2 As regards the assumptions of Theorem 2, (i) for $p>1,0<\|f\|_{p, \Phi_{-1}},\|a\|_{q, \widehat{\Psi}}<$ $\infty$, we have the following equivalent inequalities:

$$
\begin{aligned}
& \sum_{n=1}^{\infty} \int_{0}^{\infty} \frac{a_{n} f(x)}{e^{\alpha\left(\frac{V_{n}-\tau_{n}}{U(x)}\right)^{\gamma}}} d x<\frac{\Gamma(\sigma / \gamma)}{\gamma \alpha^{\sigma / \gamma}}\|f\|_{p, \Phi_{-1}}\|a\|_{q, \widehat{\Psi}}, \\
& \sum_{n=1}^{\infty} \frac{v_{n}}{\left(V_{n}-\tau_{n}\right)^{1-p \sigma}}\left[\int_{0}^{\infty} \frac{f(x)}{e^{\alpha\left(\frac{V_{n}-\tau_{n}}{U(x)}\right) \gamma}} d x\right]^{p}<\frac{\Gamma(\sigma / \gamma)}{\gamma \alpha^{\sigma / \gamma}}\|f\|_{p, \Phi_{-1}},
\end{aligned}
$$




$$
\left\{\int_{0}^{\infty} \frac{\mu(x)}{U^{1+q \sigma}(x)}\left[\sum_{n=1}^{\infty} \frac{a_{n}}{e^{\alpha\left(\frac{V_{n}-\tau_{n}}{U(x)}\right)^{\gamma}}}\right]^{q} d x\right\}^{\frac{1}{q}}<\frac{\Gamma(\sigma / \gamma)}{\gamma \alpha^{\sigma / \gamma}}\|a\|_{q, \widehat{\Psi}} ;
$$

(ii) for $p<0,0<\|f\|_{p, \Phi_{-1}},\|a\|_{q, \widehat{\Psi}}<\infty$, we have the following equivalent inequalities:

$$
\begin{aligned}
& \sum_{n=1}^{\infty} \int_{0}^{\infty} \frac{a_{n} f(x)}{e^{\alpha\left(\frac{V_{n}-\tau_{n}}{U(x)}\right)^{\gamma}}} d x>\frac{\Gamma(\sigma / \gamma)}{\gamma \alpha^{\sigma / \gamma}}\|f\|_{p, \Phi_{-1}}\|a\|_{q, \widehat{\Psi}} \\
& \sum_{n=1}^{\infty} \frac{v_{n}}{\left(V_{n}-\tau_{n}\right)^{1-p \sigma}}\left[\int_{0}^{\infty} \frac{f(x)}{e^{\alpha\left(\frac{V_{n}-\tau_{n}}{U(x)}\right)^{\gamma}}} d x\right]^{p}>\frac{\Gamma(\sigma / \gamma)}{\gamma \alpha^{\sigma / \gamma}}\|f\|_{p, \Phi_{-1}}, \\
& \left\{\int_{0}^{\infty} \frac{\mu(x)}{U^{1+q \sigma}(x)}\left[\sum_{n=1}^{\infty} \frac{a_{n}}{e^{\alpha\left(\frac{V_{n}-\tau_{n}}{U(x)}\right)^{\gamma}}}\right]^{q} d x\right\}^{\frac{1}{q}}>\frac{\Gamma(\sigma / \gamma)}{\gamma \alpha^{\sigma / \gamma}}\|a\|_{q, \widehat{\Psi}} ;
\end{aligned}
$$

(iii) for $0<p<1,0<\|f\|_{p, \Phi_{-1}},\|a\|_{q, \widehat{\Psi}}<\infty$, we have the following equivalent inequalities:

$$
\begin{aligned}
& \sum_{n=1}^{\infty} \int_{0}^{\infty} \frac{a_{n} f(x)}{e^{\alpha\left(\frac{V_{n}-\tau_{n}}{U(x)}\right)^{\gamma}}} d x>\frac{\Gamma(\sigma / \gamma)}{\gamma \alpha^{\sigma / \gamma}}\|f\|_{p, \widetilde{\Phi}_{-1}}\|a\|_{q, \widehat{\Psi}} \\
& \sum_{n=1}^{\infty} \frac{v_{n}}{\left(V_{n}-\tau_{n}\right)^{1-p \sigma}}\left[\int_{0}^{\infty} \frac{f(x)}{e^{\alpha\left(\frac{V_{n}-\tau_{n}}{U(x)}\right)^{\gamma}}} d x\right]^{p}>\frac{\Gamma(\sigma / \gamma)}{\gamma \alpha^{\sigma / \gamma}}\|f\|_{p, \widetilde{\Phi}_{-1}}, \\
& \left\{\int_{0}^{\infty} \frac{\left(1-\theta_{-1}(\sigma, x)\right)^{1-q} \mu(x)}{U^{1+q \sigma}(x)}\left[\sum_{n=1}^{\infty} \frac{a_{n}}{e^{\alpha\left(\frac{V_{n}-\tau_{n}}{U(x)}\right)^{\gamma}}}\right]^{q} d x\right\}^{\frac{1}{q}} \\
& >\frac{\Gamma(\sigma / \gamma)}{\gamma \alpha^{\sigma / \gamma}}\|a\|_{q, \widehat{\Psi}} .
\end{aligned}
$$

The above inequalities are with the best possible constant factor $\frac{\Gamma(\sigma / \gamma)}{\gamma \alpha^{\sigma / \gamma}}$.

Remark 2 (i) For $\tau_{n}=0(n \in \mathbf{N})$ in (21), setting $\Psi(n):=\frac{V_{n}^{q(1-\sigma)-1}}{v_{n}^{q-1}}(n \in \mathbf{N})$, we have the following inequality:

$$
\sum_{n=1}^{\infty} \int_{0}^{\infty} \frac{a_{n} f(x)}{e^{\alpha\left(U^{\delta}(x) V_{n}\right)^{\gamma}}} d x<\frac{\Gamma(\sigma / \gamma)}{\gamma \alpha^{\sigma / \gamma}}\|f\|_{p, \Phi_{\delta}}\|a\|_{q, \Psi}
$$

Hence, (21) is a more accurate inequality of (70) for $0<\tau_{n} \leq \frac{v_{n}}{2}$.

(ii) For $\mu(x)=v_{n}=1$ in (21), setting $0 \leq \tau \leq \frac{1}{2}$, we have the following inequality with the best possible constant factor $\frac{\Gamma(\sigma / \gamma)}{\gamma \alpha^{\sigma / \gamma}}$ :

$$
\begin{aligned}
& \sum_{n=1}^{\infty} \int_{0}^{\infty} \frac{a_{n} f(x)}{e^{\alpha\left[x^{\delta}(n-\tau)\right]^{\gamma}}} d x \\
& \quad<\frac{\Gamma(\sigma / \gamma)}{\gamma \alpha^{\sigma / \gamma}}\left[\int_{0}^{\infty} x^{p(1-\delta \sigma)-1} f^{p}(x) d x\right]^{\frac{1}{p}}\left[\sum_{n=1}^{\infty}(n-\tau)^{q(1-\sigma)-1} a_{n}^{q}\right]^{\frac{1}{q}} .
\end{aligned}
$$


In particular, for $\delta=1$, we have the following inequality with the non-homogeneous kernel:

$$
\begin{aligned}
& \sum_{n=1}^{\infty} \int_{0}^{\infty} \frac{a_{n} f(x)}{e^{\alpha[x(n-\tau)]^{\gamma}}} d x \\
& \quad<\frac{\Gamma(\sigma / \gamma)}{\gamma \alpha^{\sigma / \gamma}}\left[\int_{0}^{\infty} x^{p(1-\sigma)-1} f^{p}(x) d x\right]^{\frac{1}{p}}\left[\sum_{n=1}^{\infty}(n-\tau)^{q(1-\sigma)-1} a_{n}^{q}\right]^{\frac{1}{q}} ;
\end{aligned}
$$

for $\delta=-1$, we have the following inequality with the homogeneous kernel of degree 0 :

$$
\begin{aligned}
& \sum_{n=1}^{\infty} \int_{0}^{\infty} \frac{a_{n} f(x)}{e^{\alpha\left(\frac{n-\tau}{x}\right)^{\gamma}} d x} \\
& \quad<\frac{\Gamma(\sigma / \gamma)}{\gamma \alpha^{\sigma / \gamma}}\left[\int_{0}^{\infty} x^{p(1+\sigma)-1} f^{p}(x) d x\right]^{\frac{1}{p}}\left[\sum_{n=1}^{\infty}(n-\tau)^{q(1-\sigma)-1} a_{n}^{q}\right]^{\frac{1}{q}} .
\end{aligned}
$$

\section{Competing interests}

The authors declare that they have no competing interests.

\section{Authors' contributions}

BY carried out the mathematical studies, participated in the sequence alignment and drafted the manuscript. JL participated in the design of the study and performed the numerical analysis. All authors read and approved the final manuscript.

\section{Acknowledgements}

This work is supported by the National Natural Science Foundation of China (No. 61370186) and the Appropriative Researching Fund for Professors and Doctors, Guangdong University of Education (No. 2015ARF25). We are grateful for their help.

\section{Received: 18 November 2015 Accepted: 21 May 2016 Published online: 21 June 2016}

\section{References}

1. Hardy, GH, Littlewood, JE, Pólya, G: Inequalities. Cambridge University Press, Cambridge (1934)

2. Mitrinović, DS, Pečarić, JE, Fink, AM: Inequalities Involving Functions and Their Integrals and Derivatives. Kluwer Academic, Boston (1991)

3. Yang, BC: Hilbert-Type Integral Inequalities. Bentham Science, Sharjah (2009)

4. Yang, BC: Discrete Hilbert-Type Inequalities. Bentham Science, Sharjah (2011)

5. Yang, BC: The Norm of Operator and Hilbert-Type Inequalities. Science Press, Beijing (2009)

6. Yang, BC: On Hilbert's integral inequality. J. Math. Anal. Appl. 220, 778-785 (1998)

7. Yang, BC: An extension of a Hardy-Hilbert-type inequality. J. Guangdong Univ. Educ. 35(3), 1-8 (2015)

8. Yang, BC, Brnetić, I, Krnić, M: Generalization of Hilbert and Hardy-Hilbert integral inequalities. Math. Inequal. Appl. 8(2), 259-272 (2005)

9. Krnić, M, Pečarić, JE: Hilbert's inequalities and their reverses. Publ. Math. (Debr.) 67(3-4), 315-331 (2005)

10. Yang, BC, Rassias, TM: On the way of weight coefficient and research for Hilbert-type inequalities. Math. Inequal. Appl. 6(4), 625-658 (2003)

11. Yang, BC, Rassias, TM: On a Hilbert-type integral inequality in the subinterval and its operator expression. Banach J. Math. Anal. 4(2), 100-110 (2010)

12. Azar, L: On some extensions of Hardy-Hilbert's inequality and applications. J. Inequal. Appl. 2009, 546829 (2009)

13. Arpad, B, Choonghong, O: Best constant for certain multilinear integral operator. J. Inequal. Appl. 2006, 28582 (2006)

14. Kuang, JC, Debnath, L: On Hilbert's type inequalities on the weighted Orlicz spaces. Pac. J. Appl. Math. 1(1), 95-103 (2007)

15. Zhong, WY: The Hilbert-type integral inequality with a homogeneous kernel of Lambda-degree. J. Inequal. Appl. 2008, 917392 (2008)

16. Hong, Y: On Hardy-Hilbert integral inequalities with some parameters. JIPAM. J. Inequal. Pure Appl. Math. 6(4), Art. 92 (2005)

17. Zhong, WY, Yang, BC: On multiple Hardy-Hilbert's integral inequality with kernel. J. Inequal. Appl. 2007, Art. ID 27962 (2007)

18. Yang, BC, Krnić, M: On the norm of a mult-dimensional Hilbert-type operator. Sarajevo J. Math. 7(20), 223-243 (2011)

19. Krnić, M, Pečarić, JE, Vuković, P: On some higher-dimensional Hilbert's and Hardy-Hilbert's type integral inequalities with parameters. Math. Inequal. Appl. 11, 701-716 (2008)

20. Krnić, M, Vuković, P: On a multidimensional version of the Hilbert-type inequality. Anal. Math. 38, 291-303 (2012) 
21. Rassias, MT, Yang, BC: On half-discrete Hilbert's inequality. Appl. Math. Comput. 220, 75-93 (2013)

22. Rassias, MT, Yang, BC: A multidimensional half-discrete Hilbert-type inequality and the Riemann zeta function. Appl. Math. Comput. 225, 263-277 (2013)

23. Rassias, MT, Yang, BC: On a multidimensional half-discrete Hilbert-type inequality related to the hyperbolic cotangent function. Appl. Math. Comput. 242, 800-813 (2014)

24. Rassias, MT, Yang, BC: On a multidimensional Hilbert-type integral inequality associated to the gamma function. Appl. Math. Comput. 249, 408-418 (2014)

25. Chen, Q, Yang, BC: A survey on the study of Hilbert-type inequalities. J. Inequal. Appl. 2015, 302 (2015)

26. Gu, ZH, Yang, BC: A Hilbert-type integral inequality in the whole plane with a non-homogeneous kernel and a few parameters. J. Inequal. Appl. 2015, 314 (2015)

27. Wang, AZ, Huang, QL, Yang, BC: A strengthened Mulholland-type inequality with parameters. J. Inequal. Appl. 2015 $329(2015)$

28. Yang, BC, Chen, Q: On a Hardy-Hilbert-type inequality with parameters. J. Inequal. Appl. 2015, 339 (2015)

29. Rassias, MT, Yang, BC: A Hilbert-type integral inequality in the whole plane related to the hyper geometric function and the beta function. J. Math. Anal. Appl. 428(2), 1286-1308 (2015)

30. Yang, BC: On a more accurate multidimensional Hilbert-type inequality with parameters. Math. Inequal. Appl. 18(2), 429-441 (2015)

31. Yang, BC: A mixed Hilbert-type inequality with a best constant factor. Int. J. Pure Appl. Math. 20(3), $319-328$ (2005)

32. Yang, BC: A half-discrete Hilbert-type inequality. J. Guangdong Univ. Educ. 31(3), 1-7 (2011)

33. Zhong, WY: A mixed Hilbert-type inequality and its equivalent forms. J. Guangdong Univ. Educ. 31(5), 18-22 (2011)

34. Zhong, JH, Yang, BC: On an extension of a more accurate Hilbert-type inequality. J. Zhejiang Univ. Sci. Ed. 35(2), 121-124 (2008)

35. Yang, BC, Chen, Q: A half-discrete Hilbert-type inequality with a homogeneous kernel and an extension. J. Inequal. Appl. 2011, 124 (2011)

36. Yang, BC: A half-discrete Hilbert-type inequality with a non-homogeneous kernel and two variables. Mediterr. J. Math. 10, 677-692 (2013)

37. Yang, BC: Two Types of Multiple Half-Discrete Hilbert-Type Inequalities. Lambert Academic Publishing, Saarbrücken (2012)

38. Yang, BC: Topics on Half-Discrete Hilbert-Type Inequalities. Lambert Academic Publishing, Saarbrücken (2013)

39. Yang, BC, Debnath, L: Half-Discrete Hilbert-Type Inequalities. World Scientific, Singapore (2014)

40. Wang, ZQ, Guo, DR: Introduction to Special Functions. Science Press, Beijing (1979)

41. Kuang, JC: Applied Inequalities. Shangdong Science Technic Press, Jinan (2004)

42. Kuang, JC: Real Analysis and Functional Analysis. Higher Education Press, Beijing (2014)

\section{Submit your manuscript to a SpringerOpen ${ }^{\circ}$ journal and benefit from:}

- Convenient online submission

Rigorous peer review

- Immediate publication on acceptance

- Open access: articles freely available online

- High visibility within the field

- Retaining the copyright to your article 